D. Andreucci · P. Bisegna · E. DiBenedetto

\title{
Homogenization and concentrated capacity for the heat equation with non-linear variational data in reticular almost disconnected structures and applications to visual transduction
}

\author{
Received: July 25, 2002 \\ Published online: August 15, 2003 - @ S Springer-Verlag 2003
}

\begin{abstract}
Out of a right, circular cylinder $\Omega_{\varepsilon}$ of height $H$ and cross-section a disc of radius $R+\sigma \varepsilon$ one removes a stack of $n \approx H / \varepsilon$ parallel, equi-spaced cylinders $C_{j}, j=1,2, \ldots, n$, each of radius $R$ and height $\nu \varepsilon$. Here $\sigma, v$ are fixed positive numbers and $\varepsilon$ is a positive parameter to be allowed to go to zero. The union of the $C_{j}$ almost fills $\Omega_{\varepsilon}$ in the sense that any two contiguous cylinders $C_{j}$ are at a mutual distance of the order of $\varepsilon$ and that the outer shell, i.e., the gap $S_{\varepsilon}=\Omega_{\varepsilon}-\Omega_{o}$ has thickness of the order of $\varepsilon$ ( $\Omega_{o}$ is obtained from $\Omega_{\varepsilon}$ by formally setting $\varepsilon=0$ ). The cylinder $\Omega_{\varepsilon}$ from which the $C_{j}$ are removed, is an almost disconnected structure, it is denoted by $\widetilde{\Omega}_{\varepsilon}$, and it arises in the mathematical theory of phototransduction.

For each $\varepsilon>0$ we consider the heat equation in the almost disconnected structure $\widetilde{\Omega}_{\varepsilon}$, for the unknown function $u_{\varepsilon}$, with variational boundary data on the faces of the removed cylinders $C_{j}$. The limit of this family of problems as $\varepsilon \rightarrow 0$ is computed by concentrating heat capacity and diffusivity on the outer shell, and by homogenizing the $u_{\varepsilon}$ within the limiting cylinder $\Omega_{o}$.

It is shown that the limiting problem consists of an interior diffusion in $\Omega_{o}$ and a boundary diffusion on the lateral boundary $S$ of $\Omega_{o}$. The interior diffusion is governed by the 2-dimensional heat equation in $\Omega_{o}$, for an interior limiting function $u$. The boundary diffusion is governed by the Laplace-Beltrami heat equation on $S$, for a boundary limiting function $u_{S}$. Moreover the exterior flux of the interior limit $u$ provides the source term for the boundary diffusion on $S$. Finally the interior limit $u$, computed on $S$ in the sense of the traces, coincides with the boundary limit $u_{S}$. As a consequence of the geometry of $\widetilde{\Omega}_{\varepsilon}$, local arguments do not suffice to prove convergence in $\Omega_{o}$, and also we have to take into account the behavior of the solution in $S_{\varepsilon}$. A key, novel idea consists in extending equi-bounded and equi-Hölder continuous functions in $\varepsilon$-dependent domains, into equi-bounded and equiHölder continuous functions in the whole $\mathbb{R}^{N}$, by means of the Kirzbraun-Pucci extension technique.
\end{abstract}

D. Andreucci: Dipartimento di Metodi e Modelli Matematici, Università di Roma La Sapienza, Via A. Scarpa 16, 00161 Roma, Italy, e-mail: andreucci @dmmm . uniroma1 . it Member of INdAM-GNFM

P. Bisegna: Dipartimento di Ingegneria Civile, Università di Roma Tor Vergata, Via del Politecnico 1, 00133, Rome, Italy, e-mail: bisegna@ing . uniroma2 . it Member of INdAM-GNFM

E. DiBenedetto: Dept. of Mathematics, Vanderbilt University, Nashville, TN 37240, USA, e-mail: em. diben@Vanderbilt.edu

Partially supported by NSF grant DMS 0100660, and by NIH grant NIH R01 GM68953-01. 
The biological origin of this problem is traced, and its application to signal transduction in the retina rod cells of vertebrates is discussed.

Mathematics Subject Classification (2000). 35B27, 35K50, 92C37

Key words. homogenization - signal transduction-concentration of capacity-disconnected structure - reticular structure

\section{Introduction}

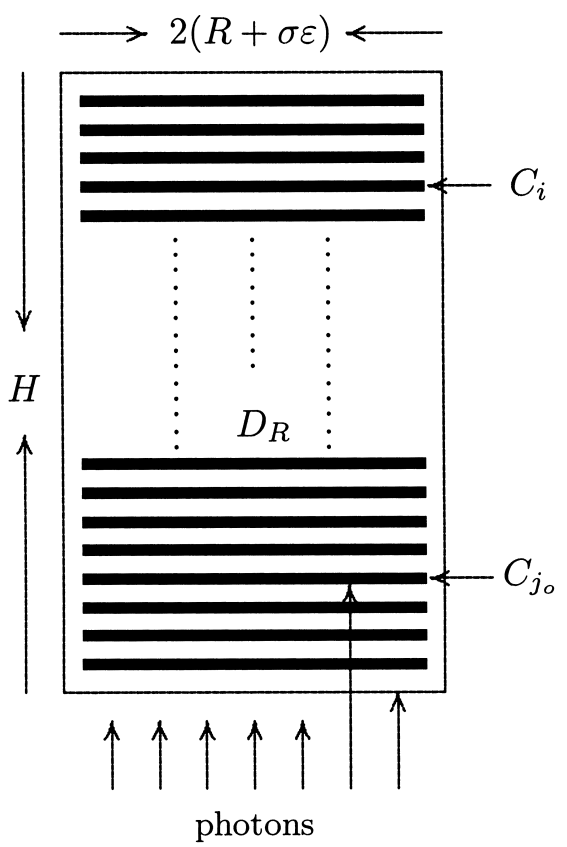

The figure represents the axial cross-section of a right circular cylinder $\Omega_{\varepsilon}$, of height $H$ and with transversal cross-section a disc $D_{R+\sigma \varepsilon}$, where $H, R$ and $\sigma$ are fixed positive numbers and $\varepsilon$ is a small positive parameter. Introduce coordinates $\bar{x}=\left(x_{1}, x_{2}\right)$ and $x=(\bar{x}, z)$ so that,

$$
\Omega_{\varepsilon}=\{|\bar{x}|<R+\sigma \varepsilon\} \times\{0<z<H\} ; \quad \Omega_{o}=\{|\bar{x}|<R\} \times\{0<z<H\} .
$$

The cylinder $\Omega_{o}$ is included in $\Omega_{\varepsilon}$, is coaxial with it, and it is formally obtained from $\Omega_{\varepsilon}$ by setting $\varepsilon=0$. The cylinder $\Omega_{o}$ houses a vertical stack of $n$ parallel, thin, equally spaced cylinders $C_{j}, j=1,2, \ldots, n$, coaxial with $\Omega_{o}$ and with crosssection a disc $D_{R}$. They are thin in the sense that their thickness is $\varepsilon \ll H$. Their mutual distance is $v \varepsilon$, where $v$ is a fixed positive number. The first $C_{1}$ has distance $\frac{1}{2} \nu \varepsilon$ from the lower face of the cylinder $\Omega_{\varepsilon}$ and the last $C_{n}$ has distance $\frac{1}{2} \nu \varepsilon$ from 
the upper face of $\Omega_{\varepsilon}$. The indicated geometry implies that,

$$
\frac{\operatorname{vol}\left(\bigcup_{j=1}^{n} C_{j}\right)}{\operatorname{vol}\left(\Omega_{o}\right)}=\frac{1}{1+v} \stackrel{\text { def }}{=} \theta_{o} ; \quad n \varepsilon=\frac{H}{1+v}=H \theta_{o} .
$$

The spaces between two contiguous cylinders $C_{j}$ and $C_{j+1}$ and within $\Omega_{o}$ are the interdiscal spaces. For $j=1,2, \ldots,(n-1)$ these are equal cylinders coaxial with $\Omega_{o}$, with the same radius and of height $\nu \varepsilon$. We label them by $I_{j}, j=0,1,2, \ldots, n$ by defining $I_{o}$ as the space between the lower face $\{z=0\}$ of $\Omega_{o}$ and the lower face of $C_{1}$, and $I_{n}$ as the space between the upper face $\{z=H\}$ of $\Omega_{o}$ and the upper face of $C_{n}$. The upper and lower faces of the interdiscal spaces $I_{j}$ are denoted by $\partial I_{j}^{ \pm}$. We also denote by $L_{j}$ the lateral surface of the discs $C_{j}$, and by $\Lambda_{j}$ the lateral surface of the interdiscal spaces $I_{j}$. The gap between $\Omega_{\varepsilon}$ and $\Omega_{o}$ is the outer shell $S_{\varepsilon}$, i.e.,

$$
S_{\varepsilon}=\{R<|\bar{x}|<R+\sigma \varepsilon\} \times\{0<z<H\} .
$$

For each of the either three-dimensional or two-dimensional domains introduced, consider the corresponding space-time cylindrical domain over a time interval $(0, T)$, for a fixed $T>0$. For example $\Omega_{o, T}=\Omega_{o} \times(0, T], S_{\varepsilon, T}=S_{\varepsilon} \times(0, T]$, etc.

\subsection{The family of $\varepsilon$-problems}

We will compute the limit $\varepsilon \rightarrow 0$ of solutions of the heat equation set in the domain $\Omega_{\varepsilon}$ from which the discs $C_{j}$ have been removed, with non-linear variational data on the faces $\partial I_{j}^{ \pm}$, and where the mass is concentrated in the outer shell $S_{\varepsilon}$. Set,

$$
a_{\varepsilon}(x)= \begin{cases}1 & \text { for } x \in \bigcup_{j=0}^{n} I_{j}, \quad \widetilde{\Omega}_{\varepsilon}=\Omega_{\varepsilon}-\bigcup_{j=1}^{n} \bar{C}_{j}=\bigcup_{j=0}^{n} I_{j} \bigcup S_{\varepsilon}, \\ \frac{\varepsilon_{o}}{\varepsilon} & \text { for } x \in S_{\varepsilon} ;\end{cases}
$$

where $\varepsilon_{o} \in(0,1)$ is fixed and $\varepsilon \in\left(0, \varepsilon_{o}\right]$. Consider the family of problems

$$
\begin{aligned}
& u_{\varepsilon} \in C\left(0, T ; L^{2}\left(\widetilde{\Omega}_{\varepsilon}\right)\right) \bigcap L^{2}\left(0, T ; W^{1,2}\left(\widetilde{\Omega}_{\varepsilon}\right)\right) \\
& a_{\varepsilon}(x) \frac{\partial}{\partial t} u_{\varepsilon}-\operatorname{div} a_{\varepsilon}(x) \nabla u_{\varepsilon}=0 \quad \text { weakly in } \widetilde{\Omega}_{\varepsilon, T},
\end{aligned}
$$

with the variational and initial data,

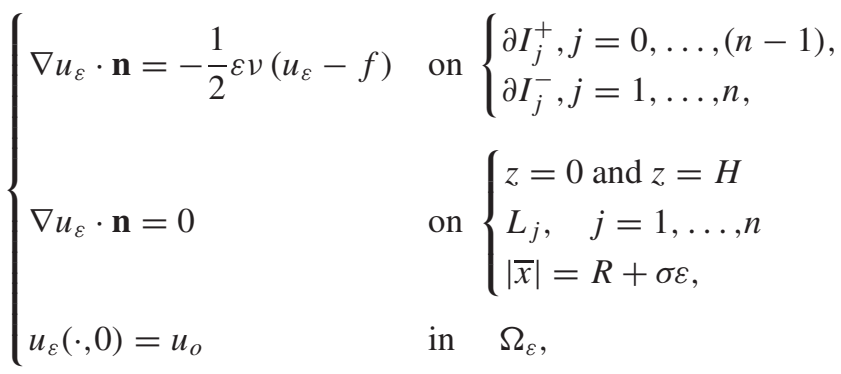


where $\mathbf{n}$ is the unit exterior normal to $\widetilde{\Omega}_{\varepsilon}$ on the indicated surfaces. The initial datum $u_{o}$ is a given positive constant. The function $f$ is the restriction to the indicated surfaces of a non-negative, bounded function with bounded gradient, defined in $\mathbb{R}^{3} \times \mathbb{R}$. The set of constants $\left\{\sigma, v, H, \varepsilon_{o}, R, T, u_{o}\right.$, sup $\left.f, \sup \left|\nabla_{x, t} f\right|\right\}$, are the data, and we say that a constant $\gamma$ depends only upon the data if it can be determined a priori only in terms of these quantities and it is independent of $\varepsilon$. The formulation (1.3) implies that $u_{\varepsilon}$ is continuous from within each of the interdiscal spaces $I_{j}$ into $S_{\varepsilon}$ through the cylindrical surfaces $\Lambda_{j}$.

\subsection{Homogenization, concentrated capacity and motivation}

For $\varepsilon=\varepsilon_{o}$ the problem (1.3) is the heat equation in $\widetilde{\Omega}_{\varepsilon}$. For $0<\varepsilon<\varepsilon_{o}$ the mass of $u_{\varepsilon}$ in the outer shell $S_{\varepsilon}$ is concentrated. Roughly speaking, the mass is divided by $\varepsilon$ to account for a shrinkage of $S_{\varepsilon}$ of the same order ([C1-9]). We will let $\varepsilon \rightarrow 0$ and $n \rightarrow \infty$ in such a way that the second of (1.1) continues to hold, i.e., the ratio between the volume of the discs and the volume of $\Omega_{o}$, remains $\theta_{o}$.

The geometry of $\widetilde{\Omega}_{\varepsilon}$ exhibits two thin compartments, available to the diffusion, i.e., the interdiscal spaces and the outer shell surrounding the stack of discs. In the limit $\widetilde{\Omega}_{\varepsilon}$ tends to $\Omega_{o}$ with no discs in it. The outer shell $S_{\varepsilon}$ tends to $S$, the lateral boundary of $\Omega_{o}$, defined by

$$
S=\{|\bar{x}|=R\} \times\{0<z<H\} .
$$

The problems in (1.3)-(1.4) tend, in a sense to be made precise, to:

i. a boundary diffusion, by the Laplace-Beltrami operator on the limiting surface $S$ (Section 2.2);

ii. a family of 2-dimensional diffusion processes, parametrized with $z \in(0, H)$ taking place on the disc $D_{R} \times\{z\}($ Section 2.1).

\section{Moreover:}

iii. the exterior fluxes of these transversal 2-dimensional diffusions serve as source terms in the boundary surface diffusion on the limiting outer shell (Section 2.2);

iv. the trace on $S$ of the solution of interior diffusion coincides with the solution of the boundary diffusion (Section 2.2).

The precise formulation of the homogenized-concentrated limit is in Sections 2-4 where we discuss its meaning, and establish its uniqueness.

This problem of homogenization-concentration is motivated by the diffusion of the second messengers cGMP (cyclic guanosin monophosphate) and $\mathrm{Ca}^{2+}$ (calcium ions) in the cytoplasm of a rod outer segment in visual transduction. A rod outer segment in the retina of vertebrates, looks like $\Omega_{\varepsilon_{o}}$. The cytoplasm is the region $\Omega_{\varepsilon_{o}}$ from which the discs $C_{j}$ have been removed. The diffusion equation (1.3)-(1.4) should be replaced by a system (for cGMP and for calcium) with somewhat more structured boundary conditions. The homogenized limiting process is suggested 
by the actual physical dimensions of $R, H$, and $\varepsilon_{o} .{ }^{1}$ The form of the fluxes in (1.4) is also generated by the physical problem, where the function $f$ is a non-linear function of calcium. Finally the problem starts from dark equilibrium, where cGMP is uniformly distributed in the rod outer segment. This motivates the assumption that $u_{o}$ is constant. The results are not affected by this assumption and $u_{o}$ could be taken as the restriction to $\widetilde{\Omega}_{\varepsilon}$ of a smooth non-negative function defined in $\mathbb{R}^{3}$.

We have chosen to present the main mathematical ideas in the context of a single equation and postpone to Appendix B a description of the visual transduction cascade generated by a photon captured by a disc $C_{i_{o}}$. There we discuss its mathematical setting and compute the corresponding homogenized-concentrated limits.

\subsection{Novelty and significance}

Limits of concentrated-capacity and homogenized limits are extensively treated in the literature in separate settings. A novelty here is their simultaneous occurrence. However the main mathematical significance of this investigation is in the novel way of computing the homogenized limit. In most of the homogenization literature the "holes" to be removed are "ball-like" and their shrinking to points does not disconnect their ancestor domain. In the homogenized geometry of $\Omega_{\varepsilon}$, the cylinders $C_{j}$ tend to discs and tend to disconnect $\Omega_{o}$. For this reason the solutions $u_{\varepsilon}$ of (1.3)-(1.4) have a topological barrier of communication between the layers of $\Omega_{\varepsilon}$. The main technical point consists of establishing that the family $\left\{u_{\varepsilon}\right\}$ is equi-Hölder continuous away from the outer shell. Since the discs essentially disconnect the rod, one needs to generate a suitable continuity estimate across the discs. What makes such an estimate possible is that $u_{\varepsilon}$ solves similar parabolic problems in neighbouring interdiscal spaces and that its mass in the outer shell has been concentrated. This is established is Sections 8-12 and it is of independent interest as it introduces novel techniques in homogenization theory. Whence such a compactness has been established, the actual computation of the homogenized limit requires that the approximating solutions be extended in some fashion with regular functions defined in the whole $\mathbb{R}^{3}$. Such an extension is realized by the Kirzbraun-Pucci theorem valid for functions with concave modulus of continuity ([G1, pp. 197-198]). This is also a novel approach to homogenization.

Homogenization procedures for structures resembling the rod are in [H4] where, however, due to homogeneous flux conditions, regularity and extensions issues are immaterial.

In Section 2 we provide a rigorous formulation of the homogenized-concentrated limit problem, in a pointwise and respectively weak form. In Section 3 we prove a uniqueness result for such a limit problem. The remaining sections are devoted to the calculation and identification of the homogenized-concentrated limit.

1 For the Salamander $H \approx 22 \mu \mathrm{m}, R \approx 5.5 \mu \mathrm{m}, \varepsilon_{o} \approx 14 \mathrm{~nm}, v \varepsilon_{o} \approx 14 \mathrm{~nm}, \sigma \varepsilon_{o} \approx$ $15 \mathrm{~nm}, n_{o} \approx 1,000 ;$ Pugh and Lamb [S5]. We refer to the review article [S6] for a detailed description of the rod anatomy. 
In Appendix A we include another way of extending the approximating solutions with functions defined in the whole rod and uniformly in a Sobolev norm. While such an extension would provide sufficient compactness to compute the homogenized limit, the Hölder continuity is of relevance in the physical problem of visual transduction. This is clarified in Appendix B (Remark B3.1), where we discuss the phototransduction cascade, set it in mathematical terms, and compute its limit.

Acknowledgement. We would like to thank R.E. Showalter for enlightening conversations and in particular for pointing out that the proof of uniqueness also contains the proof that $u_{t} \in L^{2}$. This implies the regularity of the solutions by a bootstrap argument.

\section{The homogenized-concentrated limit}

As $\varepsilon \rightarrow 0$ the family of problems (1.3)-(1.4) tends, in a sense to be made precise, to a problem involving two limiting functions

$$
\begin{aligned}
& u \text {, defined in } \Omega_{o, T} \text { called the interior limit; } \\
& \hat{u} \text {, defined in } S_{T} \text { called the limit on the outer shell. }
\end{aligned}
$$

\subsection{The interior limit}

$$
\begin{aligned}
& u \in C\left(0, T ; L^{2}\left(\Omega_{o}\right)\right),\left|\nabla_{\bar{x}} u\right| \in L^{2}\left(\Omega_{o, T}\right) ; \quad\left(\Delta_{\bar{x}}=\frac{\partial^{2}}{\partial x_{1}^{2}}+\frac{\partial^{2}}{\partial x_{2}^{2}}\right) . \\
& u_{t}-\Delta_{\bar{x}} u=-(u-f) \text { weakly in } \Omega_{o, T} ;
\end{aligned}
$$

These are diffusion processes, parametrized with $z \in(0, H)$, taking place on the disc $\{|\bar{x}|<R\}$. Also, the homogenized limit transforms the boundary fluxes in (1.4) into source terms holding in $\Omega_{o}$.

\subsection{The limit in outer shell}

Denote coordinates on the limit surface $S$ by $\theta \in(0,2 \pi]$ and $z \in(0, H)$. The level $z$ traces on $S$ a circle $\ell_{z}=\{|\bar{x}|=R\} \times\{z\}$. The restrictions of $\left\{u_{\varepsilon}\right\}$ to the outer shell $S_{\varepsilon}$ converge to a function $\hat{u}$ defined in $S_{T}$ and satisfying

$$
\hat{u} \in C\left(0, T ; L^{2}(S)\right),\left|\left(\hat{u}_{z}, \hat{u}_{\theta}\right)\right| \in L^{2}\left(S_{T}\right) .
$$

These functions are related to the interior limit $u$ as follows. First by virtue of (2.1) the function $u$ has a trace on $\{|\bar{x}|=R\}$, and such traces are in $L^{2}\left(S_{T}\right)$. Then,

$$
\hat{u}(\theta, z, t)=\left.u(\bar{x}, z, t)\right|_{|\bar{x}|=R} \quad \text { in } L^{2}\left(\ell_{z, T}\right) \quad \text { for all } z \in(0, H) .
$$

Next, denoting by $\Delta_{S}$ the Laplace-Beltrami operator on the limiting surface $S$, and by $\rho$ the radial variable on $D_{R}$,

$$
\begin{array}{ll}
\hat{u}_{t}-\Delta_{S} \hat{u}=-\left.\frac{\left(1-\theta_{o}\right)}{\sigma \varepsilon_{o}} u_{\rho}\right|_{|\bar{x}|=R} & \text { weakly in } S_{T} \\
\hat{u}_{z}(\theta, 0, t)=\hat{u}_{z}(\theta, H, t)=0 & \text { for all } t \in(0, T) \text { and } \theta \in[0,2 \pi) .
\end{array}
$$


The regularity requirement (2.1) is not sufficient to insure that $u_{\rho}$ has a trace in $L^{1}\left(S_{T}\right)$. In Section 6 we establish that (2.1) and (2.4) are meant in a classical sense and that the solution of (2.1)-(2.4) is unique.

\subsection{Weak form of the homogenized limit}

The following is the equivalent, weak form of (2.1)-(2.4). The functions $u$ and $\hat{u}$ are in the regularity classes $(2.1),(2.2)$ and satisfy,

$$
\begin{gathered}
\left(1-\theta_{o}\right)\left\{\int_{\Omega_{o, T}}\left\{-u \varphi_{t}+\nabla_{\bar{x}} u \cdot \nabla_{\bar{x}} \varphi+(u-f) \varphi\right\} d x d t\right. \\
\left.-\int_{\Omega_{o}} u_{o} \varphi(x, 0) d x\right\}_{\text {interior }} \\
+\sigma \varepsilon_{o}\left\{\int_{S_{T}}\left\{-\hat{u} \varphi_{t}+\nabla_{S} \hat{u} \cdot \nabla_{S} \varphi\right\} d \eta d t-\int_{S} u_{o} \varphi(x, 0) d \eta\right\}_{\text {outer shell }}=0,
\end{gathered}
$$

for all testing functions $\varphi \in C^{1}\left(\bar{\Omega}_{o, T}\right)$ vanishing for $t=T$. Here $\nabla_{S}$ is the gradient on $S$ and $d \eta$ is the surface measure on $S$.

The homogenized limit will be established in the weak form (2.5). In Section 6 we also establish that it is unique.

\section{Compactness}

Proposition 3.1. Let $u_{\varepsilon}$ be a solution of (1.3)-(1.4) and denote by $\gamma$ a constant depending only upon the data and independent of $\varepsilon$.

$$
\begin{aligned}
& 0 \leq u_{\varepsilon}(x, t) \leq \gamma, \quad \text { for all }(x, t) \in \widetilde{\Omega}_{\varepsilon, T} ; \\
& \sup _{0 \leq t \leq T}\left\|\sqrt{a_{\varepsilon}} u_{\varepsilon}(\cdot, t)\right\|_{2, \widetilde{\Omega}_{\varepsilon}}+\left\|\sqrt{a_{\varepsilon}} \nabla u_{\varepsilon}\right\|_{2, \widetilde{\Omega}_{\varepsilon, T}} \leq \gamma ; \\
& \int_{0}^{T-h} \int_{\widetilde{\Omega}_{\varepsilon}} a_{\varepsilon}\left[u_{\varepsilon}(t+h)-u_{\varepsilon}(t)\right]^{2} d x d t \leq \gamma h, \quad \text { for all } h \in(0, T) .
\end{aligned}
$$

Since $u_{\varepsilon, z}=0$ for $z=0$ and $z=H$, by a periodic even reflection, (1.3)(1.4) can be regarded as set in the infinite cylinder $D_{R+\sigma \varepsilon} \times \mathbb{R}$ from which one removes a periodically layered sequence of equal discs $\left\{C_{j}\right\}$. By the same token, after redefining $I_{o}$ and $I_{n}$, the interdiscal spaces $\left\{I_{j}\right\}$ form a periodically layered sequence of equal cylinders.

Proposition 3.2. Let $I_{i}$ be a fixed interdiscal space and let $\left\{z=\zeta_{2 i}\right\}$ and $\{z=$ $\left.\zeta_{2 i+1}\right\}$ be the planes containing the faces $\partial I_{i}^{ \pm}$. For $0<\delta \ll 1$ set

$$
I(\delta)=\{|\bar{x}| \leq(1-\delta) R\} \times\left(\zeta_{2 i}, \zeta_{2 i+1}\right) ; \quad I(\delta, T)=I(\delta) \times(0, T] .
$$

There exists a constant $\gamma$ independent of $\varepsilon$ and $\delta$, such that,

$$
\sup _{I(\delta, T)}\left\{\left|\nabla u_{\varepsilon}\right|+\left|u_{\varepsilon, t}\right|\right\} \leq \frac{\gamma}{\delta^{2}} .
$$


Proposition 3.3. Fix two distinct interdiscal spaces $I_{i}$ and $I_{j}$ and let $h$ be such that $I_{j}=(0,0, h)+I_{i}$. For a fixed $\delta \in(0,1)$ let $I(\delta, T)$ be defined as in (3.4). There exists a constant $\alpha \in(0,1)$ depending only upon the data and independent of $\varepsilon, h, \delta$, and a positive constant $\gamma(\delta)$ depending only upon the data and $\delta$ and independent of $h$ and $\varepsilon$, such that

$$
\sup _{I(\delta, T)}\left|u_{\varepsilon}(\bar{x}, z, t)-u_{\varepsilon}(\bar{x}, z+h, t)\right| \leq \gamma|h|^{\alpha} .
$$

Next we compute the homogenized-concentrated limit of (1.3)-(1.4), by assuming Propositions 3.1-3, whose proof is postponed to Sections 7-12.

\section{The interior limit}

In writing the weak formulation of (1.3)-(1.4) within $\Omega_{o, T}$, fix $\delta \in(0,1)$ and take testing functions $\varphi \in C_{o}^{\infty}\left(\Omega_{o, T}\right), \varphi(\cdot, 0)=0, \varphi(\cdot, T)=0$, and such that

$$
\bar{x} \longrightarrow \varphi(\bar{x}, z, t) \in C_{o}^{\infty}(\{|\bar{x}|<(1-\delta) R\}) \quad \text { for all } z \in(0, H), t \in(0, T] .
$$

Within such a domain $a_{\varepsilon} \equiv 1$. Taking into account the variational boundary data (1.4),

$$
\begin{aligned}
& \sum_{j=0}^{n} \int_{0}^{T} \int_{I_{j}}\left\{-u_{\varepsilon} \varphi_{t}-u_{\varepsilon} \Delta_{\bar{x}} \varphi\right\} d x d t+\sum_{j=0}^{n} \int_{0}^{T} \int_{I_{j}} u_{\varepsilon, z} \varphi_{z} d x d t \\
& =-\frac{1}{2} \nu \varepsilon \sum_{j=1}^{n} \int_{0}^{T} \int_{\partial I_{j}^{-}}\left(u_{\varepsilon}-f\right) \varphi d \bar{x} d t-\frac{1}{2} \nu \varepsilon \sum_{j=0}^{n-1} \int_{0}^{T} \int_{\partial I_{j}^{+}}\left(u_{\varepsilon}-f\right) \varphi d \bar{x} d t .
\end{aligned}
$$

For all $j=1, \ldots, n$,

$$
\begin{aligned}
v \varepsilon \int_{0}^{T} \int_{\partial I_{j}^{-}}( & \left.u_{\varepsilon}-f\right) \varphi d \bar{x} d t \\
& =\int_{0}^{T} \int_{I_{j}}\left(u_{\varepsilon}-f\right) \varphi d x d t-\int_{0}^{T} \int_{I_{j}}\left(\zeta_{2 j+1}-z\right)\left\{\left(u_{\varepsilon}-f\right) \varphi\right\}_{z} d x d t .
\end{aligned}
$$

By the energy estimates (3.2), the last term on right-hand side are infinitesimal of the order of $O(\varepsilon)$ and remain so even after we add them for $j=1, \ldots, n$. A similar formula holds for the last integral on the right-hand side of (4.2). Therefore (4.2) takes the form

$$
\begin{aligned}
\iint_{\Omega_{o, T}}\left\{-u_{\varepsilon} \varphi_{t}-u_{\varepsilon} \Delta_{\bar{x}} \varphi\right\} & \sum_{j=0}^{n} \chi_{I_{j}} d x d t+\iint_{\Omega_{o, T}} u_{\varepsilon, z} \varphi_{z} \sum_{j=0}^{n} \chi_{I_{j}} d x d t \\
& =-\iint_{\Omega_{o, T}}\left(u_{\varepsilon}-f\right) \varphi \sum_{j=0}^{n} \chi_{I_{j}} d x d t+O(\varepsilon) .
\end{aligned}
$$




\subsection{Extending $u_{\varepsilon}$ by equi-continuity}

Having fixed $\delta \in(0,1)$, by virtue of Propositions 3.2, 3.3 the functions $\left\{u_{\varepsilon}\right\}$ are bounded and Hölder continuous in $\widetilde{\Omega}_{\varepsilon, T} \bigcap\{|\bar{x}|<(1-\delta) R\}$, with uniform upper bound, and uniform Hölder constant and exponent. By the Kirzbraun-Pucci extension theorem ([G1, pp. 197-198]) each $u_{\varepsilon}$ can be extended with a function $\bar{u}_{\varepsilon}$ defined in the whole $\mathbb{R}^{N+1}$ with the same bounds and the same modulus of continuity. Therefore the net $\left\{\bar{u}_{\varepsilon}\right\}$ of the extensions of the $\left\{u_{\varepsilon}\right\}$ is equi-bounded and equi-Hölder continuous in $\mathbb{R}^{N+1}$.

We may let $\delta \rightarrow 0$ along a countable sequence and choose by the theorem of Ascoli-Arzelà and a diagonalization procedure, a sequence $\left\{\bar{u}_{\varepsilon_{n}}\right\}$ such that

$$
\left\{\bar{u}_{\varepsilon_{n}}\right\} \rightarrow u \quad \text { pointwise in } \Omega_{o, T} \text { and uniformly on compact subsets of } \Omega_{o, T} .
$$

In view of the uniqueness of Section 6.1, the selection of subsequences is immaterial and we will continue to label by $\varepsilon$ the various selected subsequences.

\subsection{Taking the limit in (4.3)}

By this extension the integral identity (4.3) can be written with $u_{\varepsilon}$ replaced by $\bar{u}_{\varepsilon}$. By the energy estimates (3.2),

$$
\left\{\bar{u}_{\varepsilon, z} \sum_{j=0}^{n} \chi_{I_{j}}\right\} \quad \text { is equi-bounded in } L^{2}\left(\Omega_{o, T}\right) .
$$

Therefore the selection of subsequences can be carried to ensure,

$$
\bar{u}_{\varepsilon, z} \sum_{j=0}^{n} \chi_{I_{j}} \longrightarrow \xi \quad \text { weakly in } L^{2}\left(\Omega_{o, T}\right)
$$

Lemma 4.1. Let $\theta_{o}$ be the ratio of the volume of the union of the discs $C_{i}$ with respect to the volume of $\Omega_{o}$ introduced in (1.1). Then

$$
\begin{array}{ll}
{[0, H] \ni z \rightarrow \sum_{j=0}^{n} \chi_{I_{j}}(z) \longrightarrow\left(1-\theta_{o}\right)} & \text { weakly in } L^{2}(0, H) ; \\
{[0, H] \ni z \rightarrow \int_{0}^{z} \sum_{j=1}^{n} \chi_{C_{j}} d \zeta \longrightarrow z \theta_{o}} & \text { uniformly in }[0, H] .
\end{array}
$$

Letting $\varepsilon \rightarrow 0$ in (4.3) gives

$$
\left(1-\theta_{o}\right) \iint_{\Omega_{o, T}}\left\{-u \varphi_{t}-u \Delta_{\bar{x}} \varphi+(u-f) \varphi\right\} d x d t+\iint_{\Omega_{o, T}} \xi \varphi_{z} d x d t=0 .
$$

Lemma 4.2. $\xi \equiv 0$. 
Proof. In (4.3) take the test function $\psi=\varphi \int_{0}^{z} \sum_{j=1}^{n} \chi_{C_{j}} d \zeta$ and let $\varepsilon \rightarrow 0$ to obtain

$$
\left(1-\theta_{o}\right) \iint_{\Omega_{o, T}}\left\{-u \varphi_{t}-u \Delta_{\bar{x}} \varphi+(u-f) \varphi\right\} z d x d t+\iint_{\Omega_{o, T}} \xi \varphi_{z} z d x d t=0 .
$$

Now write (4.4) with $\varphi$ replaced by $\varphi z$. Subtracting the expression so obtained from (4.4)' gives

$$
\iint_{\Omega_{o, T}} \xi \varphi d x d t=0 \quad \text { for all } \varphi \in C_{o}^{\infty}\left(\Omega_{o, T}\right) .
$$

Remark 4.1. The proof of Lemma 4.2 follows [H4] with a different technical handling.

These arguments establish the form of the interior limit as stated in Section 2.1.

\section{The global limit}

In the weak formulation of (1.3)-(1.4) we now take testing functions $\varphi \in$ $C^{1}\left(\mathbb{R}^{3} \times \mathbb{R}\right)$, vanishing for $t=T$. Write down the weak formulation and divide the various resulting integrals into the domains where the coefficients $a_{\varepsilon}$ are constant, i.e.,

$$
\begin{aligned}
& \left\{\begin{array}{c}
\int_{\widetilde{\Omega}_{\varepsilon, T}-S_{\varepsilon, T}}\left\{-u_{\varepsilon} \varphi_{t}+\nabla u_{\varepsilon} \cdot \nabla \varphi\right\} d x d t-\int_{\widetilde{\Omega}_{\varepsilon}-S_{\varepsilon}} u_{o} \varphi(x, 0) d x \\
+\frac{1}{2} \nu \varepsilon \sum_{j=0}^{n-1} \int_{0}^{T} \int_{\partial I_{j}^{+}}\left(u_{\varepsilon}-f\right) \varphi d \bar{x} d t+\frac{1}{2} \nu \varepsilon \sum_{j=1}^{n} \int_{0}^{T} \int_{\partial I_{j}^{-}}\left(u_{\varepsilon}-f\right) \varphi d \bar{x} d t
\end{array}\right\}_{\text {interior }} \\
& +\left\{\frac{\varepsilon_{o}}{\varepsilon} \iint_{S_{\varepsilon, T}}\left\{-u_{\varepsilon} \varphi_{t}+\nabla u_{\varepsilon} \cdot \nabla \varphi\right\} d x d t-\frac{\varepsilon_{o}}{\varepsilon} \int_{S_{\varepsilon}} u_{o} \varphi(x, 0) d x\right\}_{\text {outer shell }}=0 .
\end{aligned}
$$

Let $\varepsilon \rightarrow 0$ and use the results of the previous section, to obtain

$$
\begin{aligned}
& \left.\left(1-\theta_{o}\right)\left\{\int_{\Omega_{o}, T}\left\{-u \varphi_{t}+\nabla_{\bar{x}} u \cdot \nabla_{\bar{x}} \varphi+(u-f) \varphi\right\} d x d t-\int_{\Omega_{o}} u_{o} \varphi(x, 0) d x\right\}\right\}_{\text {interior }} \\
& +\lim _{\varepsilon \rightarrow 0}\left\{\frac{\varepsilon_{o}}{\varepsilon} \iint_{S_{\varepsilon, T}}\left\{-u_{\varepsilon} \varphi_{t}+\nabla u_{\varepsilon} \cdot \nabla \varphi\right\} d x d t-\frac{\varepsilon_{o}}{\varepsilon} \int_{S_{\varepsilon}} u_{o} \varphi(x, 0) d x\right\} \\
& =0 .
\end{aligned}
$$

Indeed the regularity claimed in (2.1) of $\nabla_{\bar{x}} u$ follows from standard arguments relying on the energy estimates (3.2). To compute the limit in (5.1), transform the integrals extended over $S_{\varepsilon}$ in cylindrical coordinates and set

$$
\hat{u}_{\varepsilon}(\theta, z, t)=\frac{1}{\sigma \varepsilon} \int_{R}^{R+\sigma \varepsilon} u(\rho \cos \theta, \rho \sin \theta, z, t) d \rho ; \quad \varphi(\theta, z, t)=\left.\varphi\right|_{|\bar{x}|=R} .
$$


Now require that for $|\bar{x}|>R$ the testing function $\varphi$ be independent of $|\bar{x}|$, so that $\nabla u_{\varepsilon} \cdot \nabla \varphi=u_{\varepsilon, \rho} \varphi_{\rho}+\frac{1}{\rho^{2}} u_{\varepsilon, \theta} \varphi_{\theta}+u_{\varepsilon, z} \varphi_{z}=\frac{1}{\rho^{2}} u_{\varepsilon, \theta} \varphi_{\theta}+u_{\varepsilon, z} \varphi_{z} \quad$ provided $\rho \geq R$.

Taking also into account that $\varphi$ is smooth we transform the terms under the limit in (5.1) as,

$$
\begin{aligned}
& \frac{\varepsilon_{o}}{\varepsilon}\left\{\iint_{S_{\varepsilon, T}}\left\{-u_{\varepsilon} \varphi_{t}+\nabla u_{\varepsilon} \cdot \nabla \varphi\right\} d x d t-\int_{S_{\varepsilon}} u_{o} \varphi(x, 0) d x\right\}_{\text {outer shell }} \\
& =\sigma \varepsilon_{o} \int_{0}^{T} \int_{0}^{H} \int_{0}^{2 \pi} \frac{1}{\sigma \varepsilon} \int_{R}^{R+\sigma \varepsilon}\left\{-u_{\varepsilon} \varphi_{t}+\left(\frac{1}{\rho^{2}} u_{\varepsilon, \theta} \varphi_{\theta}+u_{\varepsilon, z} \varphi_{z}\right)\right\} \rho d \rho d \theta d z d t \\
& \text { (5.2) }-\sigma \varepsilon_{o} \int_{0}^{H} \int_{0}^{2 \pi} \frac{1}{\sigma \varepsilon} \int_{R}^{R+\sigma \varepsilon} u_{o} \varphi \rho d \rho d \theta d z \\
& =\sigma \varepsilon_{o} \int_{0}^{T} \int_{0}^{H} \int_{0}^{2 \pi}\left\{-\hat{u}_{\varepsilon} \varphi_{t}+\nabla_{S} \hat{u}_{\varepsilon} \cdot \nabla_{S} \varphi\right\} R d \theta d z d t \\
& -\sigma \varepsilon_{o} \int_{0}^{H} \int_{0}^{2 \pi} u_{o} \varphi R d \theta d z+O(\varepsilon) .
\end{aligned}
$$

By the energy estimates (3.2), the nets $\left\{\hat{u}_{\varepsilon}\right\}$ and $\left\{\nabla_{S} \hat{u}_{\varepsilon}\right\}$ are equi-bounded in $L^{2}\left(S_{T}\right)$. Moreover, also taking into account the uniform time-regularity estimates in (3.3), the net $\left\{\hat{u}_{\varepsilon}\right\}$ is pre-compact in $L^{2}\left(S_{T}\right)$. Therefore for subnets relabelled with $\varepsilon$,

$$
\left\{\hat{u}_{\varepsilon}\right\} \rightarrow \hat{u} \text { strongly in } L^{2}\left(S_{T}\right) \quad \text { and } \quad\left\{\nabla_{S} \hat{u}_{\varepsilon}\right\} \rightarrow \nabla_{S} \hat{u} \text { weakly in } L^{2}\left(S_{T}\right) .
$$

Letting $\varepsilon \rightarrow 0$ in (5.2) and recalling that $R d \theta d z$ is the surface measure on $S$,

$$
\begin{aligned}
\lim _{\varepsilon \rightarrow 0} & \frac{\varepsilon_{o}}{\varepsilon}\left\{\iint_{S_{\varepsilon, T}}\left\{-u_{\varepsilon} \varphi_{t}+\nabla u_{\varepsilon} \cdot \nabla \varphi\right\} d x d t-\int_{S_{\varepsilon}} u_{o} \varphi(x, 0) d x\right\}_{\text {outer shell }} \\
= & \sigma \varepsilon_{o}\left\{\int_{S_{T}}\left\{-\hat{u} \varphi_{t}+\nabla_{S} \hat{u} \cdot \nabla_{S} \varphi\right\} d \eta d t-\int_{S} u_{o} \varphi(x, 0) d \eta\right\}_{\text {outer shell }}
\end{aligned} .
$$

Putting this in (5.1) establishes the homogenized-concentrated limit in the weak form (2.5). Finally the trace identification in (2.3) follows from the energy estimate (3.2) and the uniform Hölder estimates of the Kirzbraun-Pucci extensions $\bar{u}_{\varepsilon}$ via the triangle inequality.

\section{Regularity and uniqueness}

The integral in (2.5) extended over $\Omega_{o, T}$, does not impose any restriction on the derivatives of $\varphi$ with respect to $z$. Therefore, up to an approximation process, the testing function $\varphi$ is not required to be regular in the variable $z$ within $\{|\bar{x}|<R\}$. The integral extended over $S_{T}$ however requires that $\varphi_{z}$ be in $L^{2}\left(S_{T}\right)$. To summarize, the testing function $\varphi$ is only required to satisfy $\varphi_{t}, \nabla_{\bar{x}} \varphi \in L^{2}\left(\Omega_{o, T}\right)$. Moreover $\varphi$ must possess traces $\hat{\varphi}$ on $S_{T}$ such that $\hat{\varphi}_{t}, \nabla_{S} \hat{\varphi} \in L^{2}\left(S_{T}\right)$. 
These remarks suggest we construct testing functions of the following form. First take a function $\hat{\varphi} \in W^{1,2}\left(S_{T}\right)$ so that its traces $(\theta, t) \rightarrow \hat{\varphi}(\theta, z, t)$ are in $L^{2}\left(\ell_{z, T}\right)$ for all $z \in(0, H)$. Then for each fixed $z \in(0, H)$ extend such traces into $\{|\bar{x}|<R\}$ with a function $(\bar{x}, t) \rightarrow \varphi(\bar{x}, z, t)$ such that $\varphi_{t}$ and $\nabla_{\bar{x}} \varphi$ are in $L^{2}\left(\Omega_{o, T}\right)$.

For $0<h<T$ let $F_{h}$ denote the Steklov time-averages of a function $F \in$ $L_{\text {loc }}^{1}\left(\mathbb{R}^{N+1}\right)$. A testing function in (2.5) could be constructed by starting with $\hat{u}_{h} \in W^{1,2}\left(S_{T-h}\right)$. Then $\hat{u}_{h}$ is extended with $u_{h}$ for $z \in(0, H)$. The starting function $\hat{u}_{h}$ and its extensions are multiplied by a smooth function of $t$ that vanishes for $t \geq T-h$.

A standard change of variables shows that (2.5) holds with $u$ replaced by their time Steklov averages $u_{h}$ and it takes the form

$$
\begin{aligned}
\left(1-\theta_{o}\right) & \left\{\int_{\Omega_{o, T}}\left\{u_{h, t} \varphi+\nabla_{\bar{x}} u_{h} \cdot \nabla_{\bar{x}} \varphi+(u-f)_{h} \varphi\right\} d x d t\right\}_{\text {interior }} \\
+\sigma \varepsilon_{o} & \left\{\int_{S_{T}}\left\{\hat{u}_{h, t} \varphi+\nabla_{S} \hat{u}_{h} \cdot \nabla_{S} \varphi\right\} d \eta d t\right\}_{\text {outer shell }}=O(h),
\end{aligned}
$$

for all testing functions $\varphi \in C^{1}\left(\bar{\Omega}_{o, T}\right)$ vanishing for $t \geq T-h$.

For a fixed $2 h \in(0, T)$ let $\theta_{h}(\cdot)$ be a smooth, non-negative function defined in $\mathbb{R}$, vanishing for $t \geq T-h$ and equal to one for $t \leq T-2 h$. Then, following the previous discussion, one could take in (6.1) the testing function $\varphi=u_{h, t} \theta_{h}$. After standard calculations and limiting processes, this implies that

$$
\left\|u_{t}\right\|_{2, \Omega_{o, T}}+\left\|\Delta_{\bar{x}} u\right\|_{2, \Omega_{o, T}}+\left\|\hat{u}_{t}\right\|_{2, S_{T}} \leq \gamma
$$

for a constant $\gamma$ dependent only upon the data. Next we take into account the explicit form of the second of (2.1), when written in polar coordinates; we employ a straightforward approximation argument as well as the estimate above, and show that

$$
\left\|u_{\rho}\right\|_{2, S_{T}}+\left\|\Delta_{S} \hat{u}\right\|_{2, S_{T}} \leq \gamma,
$$

so that $u_{x_{i} x_{j}} \in L^{2}\left(\Omega_{o, T}\right)$. By a bootstrap argument and classical Schauder estimates one finds that $u$ is as regular as permitted by $f$. Thus (2.4) has a strong pointwise meaning.

\subsection{Uniqueness}

Proposition 6.1. The solution of (2.1)-(2.4) in its weak form (2.5) is unique.

Proof. Let $u_{i}, \hat{u}_{i}$ for $i=1,2$ be solutions of (2.1)-(2.4) originating from the same initial data $u_{o}$. Write (6.1) for $h=0$ and for $u_{i}$, and subtract them to obtain an integral identity for $\left(u_{1}-u_{2}\right)$. In this take the testing function $\left(u_{1}-u_{2}\right)$, which is admissible in view of the previous discussion. 


\section{Proof of Proposition 3.1}

The positivity stated in (3.1) follows from a weak form of the maximum principle. One multiplies (1.3) by $-u_{\varepsilon}^{-}$, up to Steklov averages, and examines the signs of the resulting integrals. This implies $u_{\varepsilon}^{-} \equiv 0$. Assuming the upper bounds (3.1), the energy estimates (3.2) and the time regularity in (3.3) are standard. To prove such an upper bound, in (1.3) take the test function $\left(u_{\varepsilon}-k\right)_{+}$, modulo Steklov averages, where $k \geq u_{o}$ is to be chosen. This gives,

$$
\begin{aligned}
\left\{\begin{array}{c}
\text { non-negative energy } \\
\text { terms for }\left(u_{\varepsilon}-k\right)_{+}
\end{array}\right\}= & -\frac{1}{2} v \varepsilon \sum_{j=1}^{n} \int_{0}^{T} \int_{\partial I_{j}^{-}}\left(u_{\varepsilon}-f\right)\left(u_{\varepsilon}-k\right)_{+} d \bar{x} d t \\
& -\frac{1}{2} v \varepsilon \sum_{j=0}^{n-1} \int_{0}^{T} \int_{\partial I_{j}^{+}}\left(u_{\varepsilon}-f\right)\left(u_{\varepsilon}-k\right)_{+} d \bar{x} d t
\end{aligned}
$$

Divide $\partial I_{j}^{ \pm}$into the two portions, $\left[\left(u_{\varepsilon}-f\right) \geq 0\right]$ and $\left[\left(u_{\varepsilon}-f\right)<0\right]$. The portion of the integrals extended over $\left[\left(u_{\varepsilon}-f\right) \geq 0\right]$ gives a non-positive contribution and is discarded. On the remaining portion, $u_{\varepsilon}<f$. Therefore if $k$ is chosen as $\max \left\{u_{o} ; \sup f\right\}$ all the terms on the right-hand side of (7.1) can be discarded. This in turn implies $u_{\varepsilon} \leq k$ a.e. in $\widetilde{\Omega}_{\varepsilon, T}$.

\section{Proof of Proposition 3.2}

Introduce the change of variables and the transformed function,

$$
\bar{x}=\bar{x}, \quad \zeta=\frac{R}{\nu \varepsilon}\left(z-\zeta_{2 i}\right) ; \quad U(\bar{x}, \zeta, t)=u_{\varepsilon}\left(\bar{x}, \zeta_{2 i}+\frac{\nu \varepsilon}{R} \zeta, t\right) .
$$

The cylinder $I_{i}$ is transformed into $I_{R}=D_{R} \times(0, R)$ and (1.3)-(1.4) is transformed in

$$
\begin{cases}U_{t}-\Delta_{\bar{x}} U-\left(\frac{R}{\nu \varepsilon}\right)^{2} U_{\zeta \zeta}=0 & \text { in } I_{R} \times(0, T] \\ U(\bar{x}, \zeta, 0)=u_{o} & \text { on } I_{R} \times\{0\} \\ \left(\frac{R}{v \varepsilon}\right)^{2} U_{\zeta}(\bar{x}, R, t)=-\frac{1}{2} R(U-f) & \text { for } \zeta=R \\ \left(\frac{R}{v \varepsilon}\right)^{2} U_{\zeta}(\bar{x}, 0, t)=\frac{1}{2} R(U-f) & \text { for } \zeta=0 .\end{cases}
$$

A solution of (8.1) is classical in $\bar{I}_{R}$, away from $|\bar{x}|=R$. Take the derivative of $U$ with respect to the variable $x_{\ell}$ for some $\ell=1,2,3,4$, where we stipulate that $x_{3}=\zeta$ and $x_{4}=t$. Multiply the equation so obtained by the testing function 
$\pm\left(U_{x_{\ell}}-k\right)_{ \pm} \varphi^{2}$, where $\varphi \in C_{o}^{1}\left(D_{R}\right)$, and integrate by parts over $I_{R} \times(0, t]$ for $t \in(0, T]$. This gives,

$$
\begin{aligned}
&\left\|\left(U_{x_{\ell}}-k\right)_{ \pm} \varphi\right\|_{2, I_{R} \times\{t\}}^{2}+2\left\|\varphi \nabla\left(U_{x_{\ell}}-k\right)_{ \pm}\right\|_{2, I_{R, t}}^{2} \\
&= \mp 4 \iint_{I_{R, t}}\left(U_{x_{\ell}}-k\right)_{ \pm} \varphi \nabla_{\bar{x}} \varphi \cdot \nabla_{\bar{x}}\left(U_{x_{\ell}}-k\right)_{ \pm} d x d t \\
& \pm 2\left(\frac{R}{v \varepsilon}\right)^{2} \int_{0}^{t} \int_{D_{R}} U_{\zeta x_{\ell}}\left(U_{x_{\ell}}-k\right)_{ \pm}(\bar{x}, R, \tau) \varphi^{2}(\bar{x}) d \bar{x} d \tau \\
& \mp 2\left(\frac{R}{v \varepsilon}\right)^{2} \int_{0}^{t} \int_{D_{R}} U_{\zeta x_{\ell}}\left(U_{x_{\ell}}-k\right)_{ \pm}(\bar{x}, 0, \tau) \varphi^{2}(\bar{x}) d \bar{x} d \tau .
\end{aligned}
$$

Assume first that $\ell=1,2,4$ and consider the integral on the right-hand side, written over $D_{R} \times\{\zeta=R\}$ and for $\left(U_{x_{\ell}}-k\right)_{+}$. Using the variational boundary conditions in (8.1), it can be majorized by

$$
R \int_{0}^{t} \int_{D_{R}}\left(U_{x_{\ell}}-k\right)_{+}(\bar{x}, R, \tau)\left(\left|f_{x_{\ell}}\right|-U_{x_{\ell}}\right) \varphi^{2} d \bar{x} d \tau
$$

Therefore if $k$ is chosen to satisfy $k \geq \sup |\nabla f|$, this integral gives a non-positive contribution and it can be discarded. Similar arguments hold for $\left(U_{x_{\ell}}-k\right)_{ \pm}(\bar{x}, 0, t)$. The result is that, if $\ell=1,2,4$, for such a choice of $k$, the last two boundary integrals on the right-hand side of (8.2) can be discarded. If $\ell=3$ so that $x_{3}=\zeta$, the first boundary integral on the right-hand side of (8.2) is transformed and estimated by means of the boundary conditions in the penultimate equation of (8.1), and it takes the form,

$$
\begin{aligned}
& \int_{0}^{t} \int_{D_{R}} U_{\zeta x_{\ell}}(\bar{x}, R, \tau)\left(2\left(\frac{R}{\nu \varepsilon}\right)^{2} U_{\zeta}-2\left(\frac{R}{\nu \varepsilon}\right)^{2} k\right)_{ \pm} \varphi^{2} d \bar{x} d \tau \\
& =\int_{0}^{t} \int_{D_{R}} U_{\zeta x_{\ell}}(\bar{x}, R, \tau)\left(-R(U-f)-2\left(\frac{R}{\nu \varepsilon}\right)^{2} k\right)_{ \pm} \varphi^{2} d \bar{x} d \tau .
\end{aligned}
$$

If $|k|>\gamma \sup (U+f)$ and $\varepsilon<\varepsilon_{o}$, this integral vanishes. The last boundary integral also vanishes for such a choice of $|k|$. The first integral on the right-hand side of (8.2) is majorized by the Schwarz inequality. The resulting inequalities are

$$
\begin{aligned}
\sup _{0 \leq t \leq T}\left\|\left(U_{x_{\ell}}-k\right)_{ \pm} \varphi\right\|_{2, I_{R} \times\{t\}}^{2} & +\left\|\varphi \nabla\left(U_{x_{\ell}}-k\right)_{ \pm}\right\|_{2, I_{R, T}}^{2} \\
& \leq \gamma \iint_{I_{R, T}}\left(U_{x_{\ell}}-k\right)_{ \pm}^{2}|\nabla \varphi|^{2} d x d t,
\end{aligned}
$$

for a constant $\gamma$ depending only upon the data. From this and a standard iterative technique,

$\sup _{I_{R, T} \cap\{|\bar{x}|<(1-2 \delta) R\}}\left|U_{x_{\ell}}\right| \leq \sup (U+f)+\frac{\gamma}{\delta^{5 / 6}}\left(\int_{0}^{T} \int_{0}^{R} \int_{|\bar{x}|<(1-\delta) R}\left|U_{x_{\ell}}\right|^{2} d x d t\right)^{1 / 2}$, 
for a constant $\gamma$ depending only upon the data and independent of $\delta$. Returning to the original coordinates,

$$
\sup _{I(2 \delta, T)}\left|u_{\varepsilon, x_{\ell}}\right| \leq k_{o}+\frac{\gamma}{\delta^{5 / 6}}\left(\frac{1}{\nu \varepsilon} \int_{0}^{T} \int_{\zeta_{2 i}}^{\zeta_{2 i+1}} \int_{|\bar{x}|<(1-\delta) R}\left|u_{\varepsilon, x_{\ell}}\right|^{2} d \bar{x} d z d t\right)^{1 / 2} .
$$

Assume first that $\ell=1,2,3$. From the equation in (1.3),

$$
\iint_{I_{i, T}}\left|\nabla u_{\varepsilon}\right|^{2} \varphi^{2} d x d t \leq \gamma \frac{\varepsilon}{\delta^{2}}
$$

for a non-negative, piecewise smooth cut-off function in $D_{R}$ such that it equals one on $\{|\bar{x}|<(1-\delta) R\}$ and such that $\left|\nabla_{\bar{x}} \varphi\right| \leq \gamma / \delta$. If $\ell=4$, multiplying (1.3) by $u_{\varepsilon, t} \varphi$, gives

$$
\begin{aligned}
\iint_{I_{i, T}}\left|u_{\varepsilon, t}\right|^{2} \varphi^{2} d x d t & \leq \frac{\gamma}{\delta^{2}} \iint_{I_{i, T}}\left|\nabla u_{\varepsilon}\right|^{2} \varphi^{2} d x d t \\
& -\frac{1}{2} \nu \varepsilon \int_{0}^{T} \int_{D_{R}} \varphi^{2} u_{\varepsilon, t}\left(u_{\varepsilon}-f\right)(\bar{x}, R, t) d \bar{x} d t \\
& -\frac{1}{2} \nu \varepsilon \int_{0}^{T} \int_{D_{R}} \varphi^{2} u_{\varepsilon, t}\left(u_{\varepsilon}-f\right)(\bar{x}, 0, t) d \bar{x} d t \\
& \leq \gamma \frac{\varepsilon}{\delta^{2}}
\end{aligned}
$$

\section{Proof of Proposition 3.3. Part I}

Having fixed $I_{i}$ and $I_{j}$ and $h$ as in the statement, set

$$
\begin{aligned}
& \bar{u}(\bar{x}, z, t)=u_{\varepsilon}(\bar{x}, z, t)-u_{\varepsilon}(\bar{x}, z+h, t) \\
& \bar{f}(\bar{x}, z, t)=f(\bar{x}, z, t)-f(\bar{x}, z+h, t)
\end{aligned} \quad \text { in } I_{i} .
$$

Then $\bar{u}$ satisfies the boundary-value problem in $I_{i, T}$

$$
\left\{\begin{array}{l}
\bar{u}_{t}-\Delta \bar{u}=0 ; \\
\bar{u}(\bar{x}, z, 0)=0 ; \\
\bar{u}_{z}\left(\bar{x}, \zeta_{2 i+1}, t\right)=-\frac{1}{2} v \varepsilon(\bar{u}-\bar{f})\left(\bar{x}, \zeta_{2 i+1}, t\right) ; \\
\bar{u}_{z}\left(\bar{x}, \zeta_{2 i}, t\right)=\frac{1}{2} v \varepsilon(\bar{u}-\bar{f})\left(\bar{x}, \zeta_{2 i}, t\right) .
\end{array}\right.
$$

This boundary-value problem does not contain the values of $\bar{u}$ on the lateral boundary $\Lambda_{i}$ of $I_{i}$. While such information is not directly available, it turns out that to establish the Hölder estimate in (3.6) it suffices to have only an estimate of such boundary values in their $L^{1}\left(\Lambda_{i, T}\right)$-average. 


\subsection{Estimating the $L^{1}\left(\Lambda_{i, T}\right)$-average of $\bar{u}$}

Lemma 9.1. There exists a constant $\gamma$ depending only upon the data and independent of $\varepsilon$ and $h$, such that,

$$
\frac{1}{\left|\Lambda_{i, T}\right|} \iint_{\Lambda_{i, T}}|\bar{u}(\bar{x}, z, t)| d \eta d t \leq \gamma \sqrt{|h|},
$$

where $d \eta$ is the surface measure on $\Lambda_{i}$.

Proof. Let $f$ be a smooth function defined in $\widetilde{\Omega}_{\varepsilon, T}$. In terms of cylindrical coordinates,

$$
f(R, \theta, z)=f(\rho, \theta, z)+\int_{\rho}^{R} f_{r}(r, \theta, z) d r \quad \text { for } \quad \rho>R .
$$

Integrating this in $d \rho$ over $\left(R, R+\frac{1}{2} \sigma \varepsilon\right)$ gives,

$$
\frac{1}{2} \sigma \varepsilon f(R, \theta, z)=\int_{R}^{R+\frac{1}{2} \sigma \varepsilon} f(\rho, \theta, z) d \rho+\int_{R}^{R+\frac{1}{2} \sigma \varepsilon} \int_{\rho}^{R} f_{r}(r, \theta, z) d r d \rho .
$$

Write this for $z \in\left(\zeta_{2 i}, \zeta_{2 i+1}\right)$ and $z+h \in\left(\zeta_{2 j}, \zeta_{2 j+1}\right)$ take the difference and integrate the absolute value of such a difference over $\Lambda_{i}$, i.e., integrate in $d \eta=$ $R d \theta d z$ for $\theta \in[0,2 \pi)$ and $z \in\left(\zeta_{2 i}, \zeta_{2 i+1}\right)$. These operations yield

$$
\begin{aligned}
& \int_{\Lambda_{i}}|f(R, \theta, z+h)-f(R, \theta, z)| d \eta \\
& \leq \frac{2 R}{\sigma \varepsilon} \int_{\zeta_{2 i}}^{\zeta_{2 i+1}} \int_{0}^{2 \pi} \int_{R}^{R+\frac{1}{2} \sigma \varepsilon}|f(\rho, \theta, z+h)-f(\rho, \theta, z)| d \rho d \theta d z \\
& +\frac{2 R}{\sigma \varepsilon} \int_{\zeta_{2 i}}^{\zeta_{2 i+1}} \int_{0}^{2 \pi} \int_{R}^{R+\frac{1}{2} \sigma \varepsilon} \int_{R}^{\rho}\left\{\left|f_{r}(r, \theta, z+h)\right|+\left|f_{r}(r, \theta, z)\right|\right\} d r d \rho d \theta d z \\
& =\frac{2 R}{\sigma \varepsilon} I_{1}+\frac{2 R}{\sigma \varepsilon} I_{2} .
\end{aligned}
$$

To estimate $I_{1}$ observe that as $\rho$ ranges over $\left(R, R+\frac{1}{2} \sigma \varepsilon\right)$ the argument of $f$ remains in the outer shell $S_{\varepsilon}$. Therefore,

$$
\begin{aligned}
I_{1} & \leq \int_{\zeta_{2 i}}^{\zeta_{2 i+1}} \int_{0}^{2 \pi} \int_{R}^{R+\frac{1}{2} \sigma \varepsilon} \int_{z}^{z+h}\left|f_{z}(\rho, \theta, \zeta)\right| d \zeta d \rho d \theta d z \\
& \leq v \sqrt{\frac{\pi \sigma}{R}} \sqrt{|h|} \varepsilon^{3 / 2}\left(\int_{S_{\varepsilon}}\left|f_{z}(\rho, \theta, \zeta)\right|^{2} d x\right)^{1 / 2} .
\end{aligned}
$$

Similarly $I_{2}$ is estimated as

$$
I_{2} \leq \sqrt{\frac{\pi \nu}{R}} \sigma^{3 / 2} \varepsilon^{2}\left(\int_{S_{\varepsilon}}\left|f_{r}(r, \theta, z)\right|^{2} d x\right)^{1 / 2} .
$$


Combining these estimations in (9.4) and dividing both sides by $\left|\Lambda_{i}\right|$ gives

$$
\frac{1}{\left|\Lambda_{i}\right|} \int_{\Lambda_{i}}|f(R, \theta, z+h)-f(R, \theta, z)| d \eta \leq \gamma(\sqrt{|h|}+\sqrt{\varepsilon})\left(\frac{1}{\varepsilon} \int_{S_{\varepsilon}}|\nabla f|^{2} d x\right)^{1 / 2} .
$$

To prove the lemma write this with $f=u_{\varepsilon}$ and integrate it in $d t$ over $(0, T]$. This gives

$$
\frac{1}{\left|\Lambda_{i, T}\right|} \iint_{\Lambda_{i, T}}|\bar{u}(R, \theta, z, t)| d \eta d t \leq \gamma(\sqrt{|h|}+\sqrt{\varepsilon})\left(\frac{1}{\varepsilon} \iint_{S_{\varepsilon, T}}\left|\nabla u_{\varepsilon}\right|^{2} d x d t\right)^{1 / 2}
$$

The conclusion now follows recalling that $\varepsilon \leq h$ and using the energy estimates (3.2) and the form (1.2) of the function $a_{\varepsilon}(\cdot)$.

\section{Proof of Proposition 3.3. Part II}

To proceed, in (9.2) perform the change of variables $z \rightarrow\left(z-\zeta_{2 i}\right)$ and continue to denote by $z$ the transformed variables and by $\bar{u}$ the transformed function. The domain $I_{i}$ is mapped into $\mathcal{I}=\{|\bar{x}|<R\} \times\{0<z<v \varepsilon\}$ and (9.2) continues to hold in $\mathcal{I}_{T}$ with the same boundary conditions. Denote by $\Lambda$ the lateral boundary of $\tau$, which is the transformed image of $\Lambda_{i}$. Fix a non-negative function $\xi_{\lambda} \in C_{o}^{\infty}(\mathcal{I})$ and consider the boundary-value problem,

$$
\left\{\begin{array}{l}
\varphi \in W^{1,2}\left(0, T ; L^{2}(\mathcal{I})\right) \bigcap L^{2}\left(0, T ; W^{1,2}(\mathcal{I})\right) \\
\varphi_{t}+\Delta \varphi=-\xi_{\lambda} ; \\
\varphi(x, T)=0 ;\left.\quad \varphi(\bar{x}, z, t)\right|_{\Lambda}=0 \\
\varphi_{z}(\bar{x}, 0, t)=\frac{1}{2} \nu \varepsilon \varphi(\bar{x}, 0, t) \\
\varphi_{z}(\bar{x}, \nu \varepsilon, t)=-\frac{1}{2} \nu \varepsilon \varphi(\bar{x}, \nu \varepsilon, t)
\end{array}\right.
$$

Remark 10.1. The solution of (10.1) is non-negative and it can be constructed by the Galerkin procedure. This gives $\varphi_{t} \in L^{2}\left(\mathcal{I}_{T}\right)$, with upper bounds depending upon the $L^{2}\left(\mathcal{I}_{T}\right)$-norm of $\xi_{\lambda}$.

Multiply (10.1) by $\bar{u}$ and integrate by parts the Laplacean of $\varphi$ over $\mathcal{I}_{T}$. Next take $\varphi$ in the weak formulation of (9.2). Adding the resulting inequalities,

$$
\begin{aligned}
& \iint_{\chi_{T}} \xi_{\lambda} \bar{u} d x d t=-\int_{0}^{T} \int_{\Lambda} \nabla \varphi \cdot \frac{\bar{x}}{R} \bar{u} d \eta d t \\
& -\frac{1}{2} \nu \varepsilon \int_{0}^{T} \int_{D_{R}}\{(\varphi \bar{f})(\bar{x}, 0, t)+(\varphi \bar{f})(\bar{x}, \nu \varepsilon, t)\} d \bar{x} d t \\
& \leq\left|\int_{0}^{T} \int_{\Lambda} \nabla \varphi \cdot \frac{\bar{x}}{R} \bar{u} d \eta d t\right|+\gamma \varepsilon \int_{0}^{T} \int_{D_{R}}\{\varphi|\bar{f}|(\bar{x}, 0, t)+\varphi|\bar{f}|(\bar{x}, \nu \varepsilon, t)\} d \bar{x} d t .
\end{aligned}
$$


These calculations are rigorous and can be justified by local regularization. The continuity of $u_{\varepsilon}$ will result from estimating the right-hand side of (10.2) independent of $\varepsilon, h$ and the choice of the $\xi_{\lambda} \in C_{o}^{\infty}(\mathcal{I})$. This is preceded by some estimations of the function $\varphi$.

\section{Estimating $\varphi$ away from the support of $\xi_{\lambda}$}

Select $\xi_{\lambda} \in C_{o}^{\infty}\left(\mathcal{I}_{T}\right)$ as an approximation of the identity at some point of the interior of $\mathcal{L}$, say, for example,

$\left(\bar{x}_{o}, z_{o}, t_{o}\right)$, where $\left|\bar{x}_{o}\right| \leq(1-\delta) R$ for some $\delta>0,0<z_{o}<v \varepsilon, 0<t_{o}<T$.

The kernels $\xi_{\lambda}$ satisfy

$$
\int_{\chi_{T}} \xi_{\lambda} d x d t=1 \quad \forall \lambda>0 \quad \text { and } \quad \lim _{\lambda \rightarrow 0} \int_{\chi_{T}} \xi_{\lambda} \psi(\bar{x}, z, t) d x d t=\psi\left(\bar{x}_{o}, z_{o}, t_{o}\right),
$$

for all continuous functions $\psi$ defined in $\mathcal{I}_{T}$. Set

$$
\mathcal{I}(\delta)=\{(1-\delta) R<|\bar{x}|<R\} \times\{0<z<\nu \varepsilon\} ; \quad I(\delta, T)=\mathcal{I}(\delta) \times(0, T] .
$$

Proposition 11.1. Let $\delta>0$ be fixed as in (11.1). There exists a constant $\gamma$ depending only upon the data and independent of $\varepsilon, \lambda, \delta$ such that

$$
\sup _{\chi(\delta, T)} \varphi \leq \frac{\gamma}{\delta^{2}} \frac{1}{\varepsilon} ; \quad 0 \leq-\left.\nabla \varphi \cdot \frac{\bar{x}}{R}\right|_{\Lambda} \leq \frac{\gamma}{\delta^{2}|\ln (1-\delta)|} \frac{1}{\varepsilon} .
$$

Proof. The problem (10.1) can be rescaled as in Section 8 into the new domain $\tau_{R}=D_{R} \times(0, R)$ with lateral boundary $\Lambda_{R}=\{|\bar{x}|=R\} \times\{0, R\}$. The solution of the transformed problem is then estimated, by an iteration technique, conducted near $\Lambda_{R}$, i.e., by using cut-off functions vanishing for $|\bar{x}|<(1-\delta) R$. After we return to the original coordinates, the resulting estimate takes the form

$$
\sup _{\chi(\delta, T)} \varphi \leq \frac{\gamma}{\delta^{2}} \frac{1}{\varepsilon} \iint_{\tau(4 \delta, T)} \varphi d x d t
$$

for a constant $\gamma$ depending only upon the data and independent of $\delta$ and $\varepsilon$. The integral on the right-hand side of (11.3) is estimated by referring back to (10.1). Integrating it over $\mathcal{I} \times(t, T)$,

$$
\begin{aligned}
\int_{\tau} \varphi(\bar{x}, z, t) d \bar{x} d z & -\int_{t}^{T} \int_{\Lambda} \nabla \varphi \cdot \frac{\bar{x}}{R} d \eta d \tau \\
& +\frac{1}{2} \nu \varepsilon \int_{t}^{T} \int_{D_{R}}\{\varphi(\bar{x}, 0, \tau)+\varphi(\bar{x}, \nu \varepsilon, \tau)\} d \bar{x} d \tau \\
& =\int_{t}^{T} \int_{\tau} \xi_{\lambda} d x d \tau \leq \iint_{\chi_{T}} \xi_{\lambda} d x d t=1 .
\end{aligned}
$$


The boundary integrals extended over $\{z=0\}$ and $\{z=\nu \varepsilon\}$ are non-negative and are discarded. Also the boundary integral extended over $\Lambda$ is non-negative since $\varphi$ is non-negative in $\mathcal{I}_{T}$ and vanishes on $\Lambda$.

Combining this with (11.3) proves the first of (11.2). The proof of the second is based on the construction of a barrier. Set

$$
w(\bar{x}, z, t)=\frac{\gamma}{\delta^{2} \ln (1-\delta)} \frac{1}{\varepsilon} \ln \frac{|\bar{x}|}{R} \quad \text { for }(1-\delta) R \leq|\bar{x}| \leq R .
$$

One verifies that $w$ is non-negative, it vanishes on $\Lambda$ and satisfies the equation in (10.1) within $\mathcal{I}(\delta, T)$. Moreover it has zero flux on the upper and lower faces of $\mathcal{I}(\delta)$. Finally we may assume that $w \geq \varphi$ on $|\bar{x}|=(1-\delta) R$, due to the first of (11.2), provided the constant $\gamma$ in the definition of $w$ is sufficiently large. Therefore by the maximum principle $\varphi \leq w$ within $\mathcal{I}(\delta, T)$. Since both vanish on $\Lambda$,

$$
0 \leq-\left.\nabla \varphi \cdot \frac{\bar{x}}{R}\right|_{\Lambda} \leq-\nabla w \cdot \frac{\bar{x}}{R}=\frac{-\gamma}{R \delta^{2} \ln (1-\delta)} \frac{1}{\varepsilon}
$$

\section{Proof of Proposition 3.3 concluded}

The first integral on the right-hand side of (10.2) is estimated by the second of (11.2) and (9.3), and gives,

$$
\left|\iint_{\Lambda_{i, T}} \nabla \varphi \cdot \frac{\bar{x}}{R} \bar{u} d \eta d t\right| \leq \frac{\gamma}{\delta^{2}|\ln (1-\delta)|} \sqrt{|h|} \quad \text { for all }|\bar{x}| \leq(1-\delta) R
$$

Of the remaining two boundary integrals we estimate only the first, by recalling that $f$ is smooth, and by referring back to (11.4). As indicated earlier, in this formula all integrals on the left-hand side are non-negative. Therefore

$$
\frac{1}{2} v \varepsilon \int_{0}^{T} \int_{D_{R}} \varphi|\bar{f}|(\bar{x}, 0, t) d \bar{x} d t \leq \gamma h
$$

for a constant $\gamma$ independent of $\varepsilon$. Combining these estimates in (10.2) and letting $\lambda \rightarrow 0$, gives

$$
\left|\bar{u}\left(\bar{x}_{o}, z_{o}, t_{o}\right)\right| \leq \frac{\gamma}{\delta^{3}} \sqrt{|h|} \quad \text { for all } \quad\left|\bar{x}_{o}\right| \leq(1-\delta) R
$$

This establishes (3.6) and concludes the proof of Proposition 3.3. 


\subsection{A variant of (12.2)}

The proof of Proposition 3.3 is now concluded again, under the weaker assumption

$$
|\bar{f}(\bar{x}, z, t)| \leq \frac{\gamma}{\delta^{2}|\ln (1-\delta)|} \sqrt{|h|} \quad \text { for all }|\bar{x}|<(1-\delta) R .
$$

As indicated earlier, the physical problem of visual transduction arises as a system, and the bounded function $f$ depends upon calcium which itself satisfies a diffusion equation. The proof of a statement paralleling Proposition 3.3 requires that (3.6) be established under the weaker assumption (12.3). The only difference is in the estimation of the two boundary integrals in (10.2) involving $|\bar{f}|$. We estimate the first, the estimation of the second being similar,

$$
\begin{aligned}
\int_{0}^{T} \int_{D_{R}} \varphi|\bar{f}|(\bar{x}, 0, t) d \bar{x} d t & =\int_{0}^{T} \int_{\{|\bar{x}|<(1-\delta) R\}} \varphi|\bar{f}|(\bar{x}, 0, t) d \bar{x} d t \\
& +\int_{0}^{T} \int_{\{(1-\delta) R \leq|\bar{x}|<R\}} \varphi|\bar{f}|(\bar{x}, 0, t) d \bar{x} d t .
\end{aligned}
$$

The second integral is estimated by the size of the domain of integration. Let $\tau \in(0,1)$, to be chosen. The inclusion

$$
\{(1-\delta) R \leq|\bar{x}| \leq R\} \subset\left\{\left(1-\delta^{\tau}\right) R \leq|\bar{x}| \leq R\right\}
$$

and the estimation (11.2) with $\delta$ replaced by $\delta^{\tau}$ gives

$$
\sup _{(1-\delta) R<|\bar{x}|<R} \varphi \leq \sup _{\left(1-\delta^{\tau}\right) R<|\bar{x}|<R} \varphi \leq \frac{\gamma}{\delta^{2 \tau}} \frac{1}{\varepsilon} .
$$

Then,

$$
\frac{1}{2} v \varepsilon \int_{0}^{T} \int_{\{(1-\delta) R \leq|\bar{x}|<R\}} \varphi|\bar{f}|(\bar{x}, 0, t) d \bar{x} d t \leq \gamma \delta^{1-2 \tau} .
$$

The first integral on the right-hand side of (12.4) is estimated by using (12.3). Therefore

$$
\varepsilon \int_{0}^{T} \int_{\{|\bar{x}|<(1-\delta) R\}} \varphi|\bar{f}|(\bar{x}, 0, t) d \bar{x} d t \leq \frac{\gamma}{\delta^{3}} \sqrt{|h|} \varepsilon \int_{0}^{T} \int_{D_{R}} \varphi(\bar{x}, 0, t) d \bar{x} d t \leq \frac{\gamma}{\delta^{3}} \sqrt{|h|},
$$

where we have used (11.4) again. Combine this in (10.2) and let $\lambda \rightarrow 0$ to obtain

$$
\left|\bar{u}\left(\bar{x}_{o}, z_{o}, t_{o}\right)\right| \leq \frac{\gamma}{\delta^{3}} \sqrt{|h|}+\gamma \delta^{1-2 \tau} \quad \text { for all } \quad\left|\bar{x}_{o}\right|<(1-\delta) R .
$$

Fix $|h|<1$ and $\delta \in(0,1)$. If $\delta \leq|h|^{1 / 12}$, then (3.6) follows from this for the choice $\tau=\frac{1}{4}$. If $\delta>|h|^{1 / 12}$ we observe that having established (12.5), for a fixed $\delta>0$, the same estimate continues to hold for any smaller $\delta$. Then choose $\delta=|h|^{1 / 12}$, so that the right-hand side is majorized by $\gamma|h|^{1 / 24}$. 
Appendix A: Extensions in $L^{2}\left(0, T ; W^{1,2}\left(\Omega_{o}\right)\right)$

A1. Extending $u_{\varepsilon}$ with norms equi-bounded in $L^{2}\left(0, T ; W^{1,2}\left(\Omega_{o}\right)\right)$

The limiting process on Sections 4-6 hinges upon extending $u_{\varepsilon}$ in $\mathbb{R}^{3} \times \mathbb{R}$ with equi-Hölder continuous functions that coincide with $u_{\varepsilon}$ on compact subsets of $\widetilde{\Omega}_{o}=\Omega_{o}-\bigcup_{j=1}^{n} \bar{C}_{j}$.

Here we propose an extension of $u_{\varepsilon}$ with functions $\bar{u}_{\varepsilon}$ equi-bounded in the norm of $L^{2}\left(0, T ; W^{1,2}\left(\Omega_{o}\right)\right)$, coinciding with $u_{\varepsilon}$ in $\widetilde{\Omega}_{o}$, and equi-Lipschitz continuous in $L^{2}\left(\Omega_{o, T}\right)$. It should be pointed out that such extension is possible in view of the energy estimates (3.2) when restricted to the outer shell $S_{\varepsilon}$. It would be, in general, false for functions merely defined in $\widetilde{\Omega}_{o}$, since this set is not connected.

Proposition A1.1. There exist functions $\bar{u}_{\varepsilon} \in L^{2}\left(0, T ; W^{1,2}\left(\Omega_{o}\right)\right)$, such that

$$
\begin{aligned}
& \bar{u}_{\varepsilon}=u_{\varepsilon} \quad \text { in } \bigcup_{j=0}^{n} I_{j} ; \quad\left\|\bar{u}_{\varepsilon}\right\|_{L^{2}\left(0, T ; W^{1,2}\left(\Omega_{o}\right)\right)} \leq \gamma ; \\
& \left\|\bar{u}_{\varepsilon}(t+h)-\bar{u}_{\varepsilon}(t)\right\|_{2, \Omega_{o, T-h}} \leq \gamma \sqrt{h} \quad \text { for all } h \in(0, T), \\
& \left\|\bar{u}_{\varepsilon}(z+h)-\bar{u}_{\varepsilon}(z)\right\|_{2, \Omega_{o, T}} \leq \gamma h \quad \text { for all } h \in(0, H),
\end{aligned}
$$

for a constant $\gamma$ depending only upon the data and independent of $\varepsilon$ and $h$.

Proof. Since $u_{\varepsilon}$ has zero flux for $z=0$ and $z=H$, by an even reflection, it can be regarded as defined in the infinite cylinder $D_{R} \times \mathbb{R}$ and satisfying (1.3) there.

Fix a disc $C_{j}$ with faces on the planes $z=\zeta_{2 j-1}$ and $z=\zeta_{2 j}$, and the interdiscal spaces $I_{j-1}$ and $I_{j}$ adjacent to $C_{j}$. A fixed $z \in\left[\zeta_{2 j-1}, \zeta_{2 j}\right]$ is reflected about $z=\zeta_{2 j-1}$, with weight $v$, to generate $\widehat{z}_{-} \in\left[\zeta_{2 j-2}, \zeta_{2 j-1}\right]$ belonging to the interdiscal space $I_{j-1}$, where $u_{\varepsilon}$ is well defined. The same $z$ is also reflected about $z=\zeta_{2 j}$, with weight $v$, to generate $\widehat{z}_{+} \in\left[\zeta_{2 j}, \zeta_{2 j+1}\right]$, where $u_{\varepsilon}$ is well defined. The extension of $u_{\varepsilon}$ in $C_{j}$ is obtained by interpolating the values of these functions at $\widehat{z}_{ \pm}$. Precisely,

$\left[\zeta_{2 j-1}, \zeta_{2 j}\right] \ni z \longrightarrow \quad \widehat{z}_{-}=\zeta_{2 j-1}-v\left(z-\zeta_{2 j-1}\right) ; \quad \widehat{z}_{+}=\zeta_{2 j}+v\left(\zeta_{2 j}-z\right)$,

and for $\zeta_{2 j-1} \leq z \leq \zeta_{2 j}$,

$$
\bar{u}_{\varepsilon}(\bar{x}, z, t)=u_{\varepsilon}\left(\bar{x}, \widehat{z}_{-}, t\right)\left(1+\frac{\zeta_{2 j-1}-z}{\varepsilon}\right)+u_{\varepsilon}\left(\bar{x}, \widehat{z}_{+}, t\right)\left(1-\frac{\zeta_{2 j}-z}{\varepsilon}\right) .
$$

One verifies that $\bar{u}_{\varepsilon}$ is well defined in $\Omega_{o, T}$ and that $\bar{u}_{\varepsilon}\left(\bar{x}, \zeta_{j}, t\right)=u_{\varepsilon}\left(\bar{x}, \zeta_{j}, t\right)$ for all $j$. Therefore $\bar{u}_{\varepsilon}$ is a continuous extension of $u_{\varepsilon}$ from $\widetilde{\Omega}_{o, T}$ into $\Omega_{o, T}$. The time regularity (A1.2) follows from the definition of the extension and the time regularity estimate in (3.3). Also, from the definitions,

$$
\left\|\nabla_{\bar{x}} \bar{u}_{\varepsilon}\right\|_{2, \Omega_{o, T}} \leq \gamma\left\|\nabla_{\bar{x}} u_{\varepsilon}\right\|_{2, \widetilde{\Omega}_{o, T}} .
$$

The second of (A1.1) follows from this, in view of the energy estimates (3.2), and from the estimate for $\bar{u}_{\varepsilon, z}$ which we prove below. 
The definition of $\bar{u}_{\varepsilon}$ implies,

$$
\begin{aligned}
\iint_{\Omega_{o, T}}\left|\bar{u}_{\varepsilon, z}\right|^{2} d x d t & \leq \gamma \iint_{\widetilde{\Omega}_{o, T}}\left|u_{\varepsilon, z}\right|^{2} d x d t \\
& +\frac{\gamma}{\varepsilon^{2}} \sum_{j=0}^{n} \iint_{I_{j, T}}\left|u_{\varepsilon}(z+(1+v) \varepsilon)-u_{\varepsilon}(z)\right|^{2} d x d t
\end{aligned}
$$

Also define $\bar{u}$ and $\bar{f}$ as in (9.1) and recall that they solve the boundary-value problem (9.2). A uniform bound for the last term in (A1.4), and the proof of (A1.3) follow from:

Proposition A1.2. There exists a constant $\gamma$ depending only upon the data and independent of $\varepsilon$ and $h$, such that

$$
\sum_{i=0}^{n} \iint_{I_{i, T}}\left|u_{\varepsilon}(z+h)-u_{\varepsilon}(z)\right|^{2} d x d t \leq \gamma h^{2}
$$

where $h$ is an integer multiple of $(1+v) \varepsilon$.

The proof is in two steps. First one estimates the $L^{2}\left(I_{i, T}\right)$-norm of $\bar{u}$ in terms of its $L^{2}\left(\Lambda_{i, T}\right)$-norm, i.e., their interior $L^{2}$-norm is estimated in terms of their boundary $L^{2}$-norm. This fact is of independent interest as it can be regarded as a form of the maximum principle in $L^{2}$, for solutions of (9.2). The second step consists of estimating the sum of these boundary integrals over $\Lambda_{i, T}$.

\section{A2. A form of the maximum principle in $L^{2}\left(I_{i, T}\right)$ for $\bar{u}$}

Lemma A2.1. There exists a constant $\gamma$ depending only upon the data and independent of $\varepsilon$ and $h$, such that for all $i$,

$$
\iint_{I_{i, T}}|\bar{u}|^{2} d x d t \leq \gamma \iint_{\Lambda_{i, T}}|\bar{u}|^{2} d \eta d t+\gamma h \varepsilon \sum_{\left\{k \mid \operatorname{dist}\left(I_{i}, I_{k}\right)<h\right\}} \int_{0}^{T} \int_{I_{k} \cup C_{k+1}} f_{\zeta}^{2} d x d t .
$$

Remark A2.1. The last term would be zero if $f$ were constant. In such a case the flux conditions in (9.2) would be a monotone function of $\bar{u}$. In this sense (A2.1) can be regarded as a form of a maximum principle in $L^{2}$.

Proof. As in Section 10, introduce the change of variables $z \rightarrow\left(z-\zeta_{2 i}\right)$ and continue to denote by $z$ the transformed variable and by $\bar{u}$ the transformed function. The domain $I_{i}$ is mapped into the cylinder $\mathcal{I}$ introduced in Section 10, the lateral boundary $\Lambda_{i}$ of $I_{i}$ is transformed in $\Lambda$, and (9.2) continues to hold in $\tau_{T}$ with the 
same boundary conditions. Introduce the problem

$(\mathrm{A} 2.2)$

$$
\left\{\begin{array}{l}
\varphi \in W^{1,2}\left(0, T ; L^{2}(\mathcal{I})\right) \cap L^{2}\left(0, T ; W^{1,2}(\mathcal{I})\right) \\
\varphi_{t}+\Delta \varphi=-\bar{u} ; \\
\varphi(x, T)=0 ;\left.\quad \varphi(\bar{x}, z, t)\right|_{\Lambda}=0 \\
\varphi_{z}(\bar{x}, 0, t)=\frac{1}{2} \nu \varepsilon \varphi(\bar{x}, 0, t) \\
\varphi_{z}(\bar{x}, \nu \varepsilon, t)=-\frac{1}{2} \nu \varepsilon \varphi(\bar{x}, \nu \varepsilon, t) .
\end{array}\right.
$$

This is the analogue of (10.1) where the role of the right-hand side $\xi_{\lambda}$ was to reproduce, as $\lambda \rightarrow 0$, the pointwise values of $\bar{u}$. The role of the right-hand side of (A2.2) is to generate the $L^{2}\left(\mathcal{I}_{T}\right)$-norm of $\bar{u}$. Multiply (A2.2) by $\bar{u}$ and integrate by parts over $\mathcal{I}_{T}$. Also use $\varphi$ as a testing function in the weak formulation of (9.2). Adding the resulting equalities,

$$
\begin{aligned}
\iint_{\chi_{T}} \bar{u}^{2} d x d t= & -\int_{0}^{T} \int_{\Lambda} \nabla \varphi \cdot \frac{\bar{x}}{R} \bar{u} d \eta d t \\
& -\frac{1}{2} \nu \varepsilon \int_{0}^{T} \int_{D_{R}}\{(\varphi \bar{f})(\bar{x}, 0, t)+(\varphi \bar{f})(\bar{x}, \nu \varepsilon, t)\} d \bar{x} d t .
\end{aligned}
$$

This provides an analogue of (10.2), i.e.,

$$
\begin{aligned}
\iint_{\mathcal{I}_{T}} \bar{u}^{2} d x d t & \leq \frac{\gamma}{\theta} \int_{0}^{T} \int_{\Lambda} \bar{u}^{2} d \eta d t+\theta \int_{0}^{T} \int_{\Lambda}\left|\nabla \varphi \cdot \frac{\bar{x}}{R}\right|^{2} d \eta d t \\
& +\gamma \varepsilon \int_{0}^{T} \int_{D_{R}}\{|\varphi \bar{f}|(\bar{x}, 0, t)+|\varphi \bar{f}|(\bar{x}, \nu \varepsilon, t)\} d \bar{x} d t,
\end{aligned}
$$

for a fixed constant $\gamma$ depending only upon the data and for a positive parameter $\theta$ to be chosen. The estimate (A1.5) will result from estimating the right-hand side of (A2.3).

Assume that $\varphi$ is of class $C^{3}$ up to $\{|\bar{x}|=R\}$ for all $0<z<v \varepsilon$. Then,

$$
\int_{\{|\bar{x}|=R\}}\left|\nabla \varphi \cdot \frac{\bar{x}}{R}\right|^{2}(\bar{x}, z, t) d \ell \leq \gamma \int_{D_{R}}\left\{|\Delta \varphi|^{2}+\left|\nabla_{\bar{x}} \varphi\right|^{2}+\varphi^{2}\right\}(\bar{x}, z, t) d \bar{x},
$$

where $d \ell$ is the line measure on $\{|\bar{x}|=R\}$. Integrate these in $d z$ over $(0, v \varepsilon)$ and in $d t$ over $(0, T)$. Using (A2.1) and standard energy estimates,

$$
\int_{0}^{T} \int_{\Lambda}\left|\nabla_{\bar{x}} \varphi \cdot \frac{\bar{x}}{R}\right|^{2} d \eta d t \leq \gamma \iint_{\chi_{T}} \bar{u}^{2} d x d t+\gamma \iint_{\chi_{T}} \varphi_{t}^{2} d x d t .
$$

The smoothness required by these inequalities, can be achieved by smoothing $\bar{u}$ in (A2.2) and by a limiting process. To estimate the last integrals on the right-hand side, multiply (A2.2) by $\varphi_{t}$, modulo a Steklov time averaging, and integrate by parts. This gives,

$$
\iint_{\chi_{T}} \varphi_{t}^{2} d x d t+\frac{1}{2} \nu \varepsilon \sup _{0<t<T} \int_{D_{R}}\left\{\varphi^{2}(\bar{x}, 0, t)+\varphi^{2}(\bar{x}, \nu \varepsilon, t)\right\} d \bar{x} \leq \iint_{\chi_{T}} \bar{u}^{2} d x d t .
$$


Combining this with (A2.4) and (A2.3), and choosing $\theta$ sufficiently small,

$$
\begin{aligned}
\iint_{x_{T}} \bar{u}^{2} d x d t \leq & \gamma \int_{0}^{T} \int_{\Lambda} \bar{u}^{2} d \eta d t \\
& -\gamma \theta \varepsilon \sup _{0<t<T} \int_{D_{R}}\left\{\varphi^{2}(\bar{x}, 0, t)+\varphi^{2}(\bar{x}, \nu \varepsilon, t)\right\} d \bar{x} \\
& +\gamma \varepsilon \int_{0}^{T} \int_{D_{R}}\{|\varphi \bar{f}|(\bar{x}, 0, t)+|\varphi \bar{f}|(\bar{x}, \nu \varepsilon, t)\} d \bar{x} d t,
\end{aligned}
$$

for a constant $\gamma$ depending only upon the data and independent of $\varepsilon$ and $h$. The last two integrals are majorized by the Cauchy-Schwarz inequality and the terms containing $\varphi^{2}$ are eliminated with the homologous one appearing negatively. Thus returning to the original coordinates,

$$
\begin{aligned}
\iint_{I_{i, T}} \bar{u}^{2} d x d t \leq & \gamma \iint_{\Lambda_{i, T}} \bar{u}^{2} d \eta d t \\
& +\gamma \varepsilon \iint_{D_{R, T}}\left\{\bar{f}^{2}\left(\bar{x}, \zeta_{2 i-1}, t\right)+\bar{f}^{2}\left(\bar{x}, \zeta_{2 i}, t\right)\right\} d \bar{x} d t .
\end{aligned}
$$

From the definition of $\bar{f}$,

$$
\begin{aligned}
\iint_{D_{R, T}} \bar{f}^{2}\left(\bar{x}, \zeta_{2 i}, t\right) d \bar{x} d t & =\iint_{D_{R, T}}\left|f\left(\bar{x}, \zeta_{2 i}+h, t\right)-f\left(\bar{x}, \zeta_{2 i}, t\right)\right|^{2} d \bar{x} d t \\
& =\int_{0}^{T} \int_{D_{R}}\left|\int_{\zeta_{2 i}}^{\zeta_{2 i}+h} f_{\zeta}(\bar{x}, \zeta, t) d \zeta\right|^{2} d \bar{x} d t \\
& \leq h \int_{0}^{T} \int_{D_{R}} \int_{\zeta_{2 i}}^{\zeta_{2 i}+h} f_{\zeta}^{2}(\bar{x}, \zeta, t) d \zeta d \bar{x} d t \\
& \leq \gamma h \sum_{\left\{k \mid \text { dist }\left\{I_{i}, I_{k}\right\}<h\right\}} \int_{0}^{T} \int_{I_{k} \cup C_{k+1}} f_{\zeta}^{2} d x d t
\end{aligned}
$$

The integral extended over $z=\zeta_{2 i-1}$ is estimated similarly.

Remark A2.2. Since $f$ is smooth, the penultimate term in (A2.6) could be estimated by $\gamma h^{2}$. We have chosen, however, the less sharp estimate (A2.1) in view of the applications of such an extension technique to the originating problem in visual transduction, where $f$ is a function of calcium and as such, possesses a lesser degree of regularity.

\section{A3. Proof of Proposition A1.2 concluded}

By calculations in all analogue to those of Section 9,

$$
\sum_{i=0}^{n} \iint_{\Lambda_{i, T}} \bar{u}^{2} d \eta d t \leq \gamma|h|^{2}\left(\frac{\varepsilon_{o}}{\varepsilon} \iint_{S_{\varepsilon, T}}\left|\nabla u_{\varepsilon}\right|^{2} d \bar{x} d z d t\right) \leq \gamma|h|^{2},
$$


by the energy estimates (3.3). Putting this in (A2.1) and adding over $i$,

$$
\sum_{i=0}^{n} \iint_{I_{i, T}} \bar{u} d x d t \leq \gamma h^{2}+\gamma \varepsilon h \sum_{i=0}^{n} \sum_{\left\{k \mid \operatorname{dist}\left(I_{i} ; I_{k}\right)<h\right\}} \int_{0}^{T} \int_{I_{k} \cup C_{k+1}} f_{z}^{2} d x d t .
$$

Now for a fixed $i$ there are, at most, $h / \varepsilon$ interdiscal spaces $I_{k} \operatorname{such}$ that $\operatorname{dist}\left(I_{i} ; I_{k}\right)$ $<h$. Therefore in the double sum with indices $i$ and $k$, the integrals extended over a fixed $I_{k}$ are repeated at most $(h / \varepsilon)$-times, and

$$
\gamma \varepsilon h \sum_{i=0}^{n} \sum_{\left\{k \mid \operatorname{dist}\left(I_{i} ; I_{k}\right)<h\right\}} \int_{0}^{T} \int_{I_{k} \cup C_{k+1}} f_{z}^{2} d x d t \leq \gamma h^{2} \iint_{\Omega_{o, T}} f_{z}^{2} d x d t .
$$

\section{Appendix B: Applications to visual transduction}

\section{B1. Diffusion of cGMP and $\mathrm{Ca}^{2+}$}

The membrane on the lateral boundary of the rod outer segment contains ionic channels. These are kept open by the presence of cGMP (cyclic guanosin monophosphate), allowing the influx of calcium ions $\mathrm{Ca}^{2+}$. A steady state is maintained by the presence of an ionic exchanger which expels calcium.

Both cGMP and $\mathrm{Ca}^{2+}$ can diffuse within the cytosol, i.e., the portion of the rod, not occupied by the discs. They cannot penetrate the discs, although cGMP can be depleted or generated by sources located on the faces of the discs.

The rod functions as a light-capturing device. As a photon hits the rod, it is captured by one of the discs, say $C_{i_{o}}$, and it triggers a biochemical cascade whose net effect is the depletion of cGMP.

When cGMP is depleted the channels close, thereby causing a drop in membrane current. Vision is modulated by these variations of ionic current on the boundary of the rods [S6].

Denote by $u_{\varepsilon_{o}}$ and $v_{\varepsilon_{o}}$ the volumic, dimensionless concentrations of cGMP and $\mathrm{Ca}^{2+}$ in the cytosol and rescale lengths and times so that the various parameters, for example $R, \varepsilon_{o}$, are all dimensionless. The functions $u_{\varepsilon_{o}}$ and $v_{\varepsilon_{o}}$ satisfy the diffusion equations,

$$
\begin{aligned}
& u_{\varepsilon_{o}, t}-k_{u} \Delta u_{\varepsilon_{o}}=0 \\
& v_{\varepsilon_{o}, t}-k_{v} \Delta v_{\varepsilon_{o}}=0
\end{aligned} \quad \text { in } \widetilde{\Omega}_{\varepsilon_{o}, T},
$$

where $k_{u}$ and $k_{v}$ are the diffusion coefficients of cGMP and $\mathrm{Ca}^{2+}$, respectively. Their non-linear coupling occurs through their fluxes on the faces $\partial I_{j}^{ \pm}$,

(B1.2) $k_{u} u_{\varepsilon_{o}, z}=\left\{\begin{array}{c}-\frac{1}{2} v \varepsilon_{o}\left\{\gamma_{o} u_{\varepsilon_{o}}-f\left(v_{\varepsilon_{o}}\right)\right\} \\ -\chi_{\left\{z=z_{o}\right\}} u_{\varepsilon_{o}} f_{1}\left(v_{\varepsilon_{o}}, x, t\right)\end{array} \quad\right.$ on $\partial I_{j}^{+}, j=0,1, \ldots,\left(n_{o}-1\right)$, 
where $\gamma_{o}$ is a given positive constant and $f$ and $f_{1}$ are given, positive, bounded, smooth functions of their arguments. The coordinate $z=z_{o}$ is that of the face $\partial I_{j_{o}}^{+}$hit by the photon (i.e., $z_{o}=\zeta_{2 j_{o}+1}$; note that $i_{o}=j_{o}+1$ ). The characteristic function $\chi_{\left\{z=z_{o}\right\}}$ permits one to account for the depletion sources, localized at $z_{o}$ and due to the action of the photon. Moreover cGMP does not penetrate the discs $C_{j}$ through their lateral boundaries $L_{j}$, nor can it exit the boundary of the rod, i.e.,

$$
\nabla u_{\varepsilon_{o}} \cdot \mathbf{n}=0 \quad \text { on } L_{j} \text {, on }|\bar{x}|=R+\sigma \varepsilon_{o} \text {, on } z=0 \text { and } z=H .
$$

Calcium $v_{\varepsilon_{o}}$ does not penetrate the $\operatorname{discs} C_{j}$, nor outflows the rod through its bottom $z=0$ or top $z=H$, i.e.,

$$
\nabla v_{\varepsilon_{o}} \cdot \mathbf{n}=0 \quad \text { on } L_{j} \text {, on } \partial I_{j}^{ \pm} \text {, on } z=0 \text { and } z=H .
$$

However, it can flow through the lateral boundary of the rod,

$$
k_{v} \nabla v_{\varepsilon_{o}} \cdot \mathbf{n}=-g_{1}\left(v_{\varepsilon_{o}}\right)+g_{2}\left(u_{\varepsilon_{o}}\right) \quad \text { on }|\bar{x}|=R+\sigma \varepsilon_{o},
$$

for given, positive, bounded, smooth functions $g_{1}(\cdot)$ and $g_{2}(\cdot)$. Here $\mathbf{n}$ is the outward unit exterior normal to $\widetilde{\Omega}_{\varepsilon_{o}}$, at the indicated surfaces. A complete description of the phototransduction cascade, as well as a detailed derivation of the model (B1.1)-(B1.5) is in [S6,1]. There, the various boundary terms are justified and discussed. In particular the functions $f, g_{1}, g_{2}$ are explicit and given by

$$
\mathbb{R}^{+} \ni s \longrightarrow f(s)=\frac{\gamma_{1}}{\beta_{1}^{m}+s^{m}} ; \quad g_{1}(s)=\frac{c_{1} s}{d_{1}+s} ; \quad g_{2}(s)=\frac{c_{2} s^{\kappa}}{d_{2}^{\kappa}+s^{\kappa}}
$$

for given positive constants $\gamma_{1}, \beta_{1}, m, c_{1}, c_{2}, d_{1}, d_{2}, \kappa$. The model in [S1] assumes that a single photon hits the rod at the disc $C_{i_{o}}$ [S5-7]. However, the form of these functions is independent of light intensity. The phenomenon starts at time $t=0$ from a dark equilibrium. It is assumed that in absence of light the system is in a constant steady state, i.e., $u_{\varepsilon_{o}}=u_{o}$ and $v_{\varepsilon_{o}}=v_{o}$ for two given positive constants $u_{o}$ and $v_{o}{ }^{2}$

\section{B1.1. Homogenization and concentrated capacity}

The geometry of the rod exhibits two thin compartments, available to the diffusion: the interdiscal spaces and the outer shell surrounding the stack of discs. The diffusion within the interdiscal spaces cannot be neglected in view of the reaction terms acting on the faces of the discs. The diffusion along the outer shell cannot be neglected because it regulates the opening and closing of the ionic channels.

\footnotetext{
${ }^{2}$ In dark equilibrium [cGMP] $=2-4 \mu \mathrm{M}$ and $\left[\mathrm{Ca}^{2+}\right]=.4 \mu \mathrm{M}$ ([S6]). Thus $u_{o} \approx 3$ and $v_{o} \approx .4$.
} 
The values $\varepsilon_{o} \ll R$ suggest the homogenization process described in Section 1.2. The only difference here is that by letting $\varepsilon \rightarrow 0$ along a suitable sequence, the face $z=z_{o}$ is kept constant. Thus $j_{o}=j_{o}(\varepsilon)$ but $\zeta_{2 j_{o}+1}=z_{o}$ for all $\varepsilon \leq \varepsilon_{o}$.

Consider the interdiscal space $I_{j_{o}}$ whose face $\partial I_{j_{o}}^{+}$is hit by the photon. This is the only physical compartment where cGMP can flow from the outer shell to the depletion sites activated by the photon. To keep the spatial localization of the activation site, the width of $I_{j_{o}}$ is sent to zero by a capacity concentration similar to the one in the outer shell.

The approximating problems encompassing both homogenization and concentration of capacities are introduced in Section B2.

The homogenized-concentrated limit has the same structure as that in Section 2, except that concentrating the mass on $I_{j_{o}}$ gives rise to a further transversal 2-dimensional diffusion on $D_{R}$ at the level $z_{o}$ both for cGMP and calcium (Section B3.2). Their exterior fluxes at $z_{o}$ serve as sources in the boundary diffusion, localized through a Dirac mass at $z_{o}$ (Section B3.3).

\section{B2. The approximating $\varepsilon$-problems}

The functions in play are $u_{\varepsilon}$ and $v_{\varepsilon}$ defined in $\widetilde{\Omega}_{\varepsilon, T}$ and representing dimensionless approximations of cGMP and $\mathrm{Ca}^{2+}$. Set

$$
a_{\varepsilon}(x)= \begin{cases}1 & \text { for } x \in \bigcup_{i \neq j_{o}} I_{j} \\ \frac{\varepsilon_{o}}{\varepsilon} & \text { for } x \in I_{j_{o}} \bigcup S_{\varepsilon}\end{cases}
$$

The families $\left\{u_{\varepsilon}\right\}$ and $\left\{v_{\varepsilon}\right\}$ satisfy,

$$
\begin{aligned}
& u_{\varepsilon}, v_{\varepsilon} \in C\left(0, T ; L^{2}\left(\widetilde{\Omega}_{\varepsilon}\right)\right) \bigcap L^{2}\left(0, T ; W^{1,2}\left(\widetilde{\Omega}_{\varepsilon}\right)\right) \\
& a_{\varepsilon}(x) \frac{\partial}{\partial t} u_{\varepsilon}-k_{u} \operatorname{div} a_{\varepsilon}(x) \nabla u_{\varepsilon}=0 \\
& a_{\varepsilon}(x) \frac{\partial}{\partial t} v_{\varepsilon}-k_{v} \operatorname{div} a_{\varepsilon}(x) \nabla v_{\varepsilon}=0,
\end{aligned}
$$

with the following variational conditions on $\partial \widetilde{\Omega}_{\varepsilon}$ :

$$
\begin{aligned}
& k_{u} u_{\varepsilon, z}=\left\{\begin{array}{cl}
-\frac{1}{2} \nu \varepsilon\left\{\gamma_{o} u_{\varepsilon}-f\left(v_{\varepsilon}\right)\right\} & \text { on } \partial I_{j}^{+}, j=0,1, \ldots,(n-1), j \neq j_{o} \\
\frac{1}{2} \nu \varepsilon\left\{\gamma_{o} u_{\varepsilon}-f\left(v_{\varepsilon}\right)\right\} & \text { on } \partial I_{j}^{-}, j=1, \ldots, n
\end{array}\right. \\
& \frac{\varepsilon_{o}}{\varepsilon} k_{u} u_{\varepsilon, z}=-\frac{1}{2} \nu \varepsilon_{o}\left\{\gamma_{o} u_{\varepsilon}-f\left(v_{\varepsilon}\right)\right\}-u_{\varepsilon} f_{1}\left(v_{\varepsilon}, x, t\right) \quad \text { on } \partial I_{j_{o}}^{+}
\end{aligned}
$$




$$
\begin{aligned}
& \nabla u_{\varepsilon} \cdot \mathbf{n}=0 \quad \text { on } \quad z=0, z=H, L_{j},|\bar{x}|=R+\sigma \varepsilon \\
& \nabla v_{\varepsilon} \cdot \mathbf{n}=0 \quad \text { on } \quad z=0, z=H, L_{j}, \partial I_{j}^{ \pm} \\
& \frac{\varepsilon_{o}}{\varepsilon} k_{v} \nabla v_{\varepsilon} \cdot \mathbf{n}=-g_{1}\left(v_{\varepsilon}\right)+g_{2}\left(u_{\varepsilon}\right) \quad \text { on } \quad|\bar{x}|=R+\sigma \varepsilon,
\end{aligned}
$$

where $\mathbf{n}$ is the unit exterior normal to $\widetilde{\Omega}_{\varepsilon}$ on the indicated surfaces. The initial conditions are those of dark equilibrium, i.e., $u_{\varepsilon}(\cdot, 0)=u_{o}$ and $v_{\varepsilon}(\cdot, 0)=v_{o}$ for two given positive constants $u_{o}, v_{o}$.

Remark B2.1. The problem (B2.2)-(B2.5) coincides with (B1.1)-(B1.5) for $\varepsilon=\varepsilon_{o}$. For $0<\varepsilon<\varepsilon_{o}$, the mass of cGMP and $\mathrm{Ca}^{2+}$ within the interdiscal space $I_{j_{o}}$ and the outer shell $S_{\varepsilon}$ is concentrated. Roughly speaking, in these domains the mass is divided by $\varepsilon$ to account for a shrinkage of the domain of the same order.

\section{B3. The homogenized-concentrated limit}

As $\varepsilon \rightarrow 0$ the family in (B2.1)-(B2.5) generates three pairs of functions $u, v$ defined in $\Omega_{o, T}$ are called the interior limit;

$\stackrel{\circ}{u}, \stackrel{\circ}{v}$ defined in $D_{R} \times\left\{z_{o}\right\}$ are called the limit on the special level $z_{o}$;

$\hat{u}, \hat{v}$ defined in $S_{T}$ are called the limit on the outer shell.

\section{B3.1. The interior limit}

$$
\begin{aligned}
& u, v \in C\left(0, T ; L^{2}\left(\Omega_{o}\right)\right) ;\left|\nabla_{\bar{x}} u\right|,\left|\nabla_{\bar{x}} v\right| \in L^{2}\left(\Omega_{o, T}\right) \\
& u, v \in C_{\mathrm{loc}}^{\alpha}\left(\Omega_{o, T}\right) \text { for some } \alpha \in(0,1) \\
& u_{t}-k_{u} \Delta_{\bar{x}} u=-\left\{\gamma_{o} u-f_{1}(v)\right\} \quad \text { weakly in } \Omega_{o, T} . \\
& v_{t}-k_{v} \Delta_{\bar{x}} v=0
\end{aligned}
$$

This is a family of diffusion processes in $D_{R}$, parametrized with $z \in(0, H)$. The boundary fluxes in (B2.3) are transformed into sources in $\Omega_{o}$.

Remark B3.1. The solutions are $C^{\infty}\left(\Omega_{o, T}\right)$ except across the hyperplane $\left\{z=\zeta_{o}\right\}$, but remain Hölder continuous across $z=z_{o}$.

B3.2. The limit on the special level $z_{o}$

$$
\begin{aligned}
\stackrel{\circ}{u}, \stackrel{\circ}{v} \in C(0, T ; & \left.L^{2}\left(D_{R} \times\left\{z_{o}\right\}\right)\right) ;\left|\nabla_{\bar{x}} \stackrel{\circ}{u}\right|,\left|\nabla_{\bar{x}} \stackrel{\circ}{v}\right| \in L^{2}\left(D_{R, T} \times\left\{z_{o}\right\}\right) ; \\
\stackrel{\circ}{u}_{t}-k_{u} \Delta_{\bar{x}} \stackrel{\circ}{u}= & -\left\{\gamma_{o} \stackrel{\circ}{u}-f_{1}(\stackrel{\circ}{v})\right\} \\
& -\frac{1}{v \varepsilon_{o}} \stackrel{\circ}{u} f_{2}\left(\stackrel{\circ}{v}, \bar{x}, z_{o}, t\right) \quad \text { weakly in } D_{R, T} \times\left\{z_{o}\right\} . \\
\stackrel{\circ}{v}_{t}-k_{v} \Delta_{\bar{x}} \stackrel{\circ}{v}= & 0 .
\end{aligned}
$$


Thus at the special level $z_{o}$ the concentrated limit is a diffusion equation holding on a disc and with sources inherited from the fluxes in (B2.3). The solutions are $C^{\infty}\left(D_{R, T}\right)$.

\section{B3.3. The limit in outer shell}

The restrictions of $\left\{u_{\varepsilon}\right\}$ and $\left\{v_{\varepsilon}\right\}$ to the outer shell $S_{\varepsilon, T}$ converge to functions $\hat{u}, \hat{v}$ defined in $S_{T}$ and satisfying

$$
\hat{u}, \hat{v} \in C\left(0, T ; L^{2}(S)\right) ; \quad\left|\left(\hat{u}_{z}, \hat{u}_{\theta}\right)\right|,\left|\left(\hat{v}_{z}, \hat{v}_{\theta}\right)\right| \in L^{2}\left(S_{T}\right) .
$$

These are related to the interior limits $u$ and $v$, and to $\stackrel{\circ}{u}$ and $\stackrel{\circ}{v}$ by sharing their traces, i.e.,

$$
\begin{array}{lll}
\hat{u}(\theta, z, t)=\left.u(\bar{x}, z, t)\right|_{|\bar{x}|=R} & \text { in } L^{2}\left(\ell_{z, T}\right), & \text { for all } z \neq z_{o} ; \\
\hat{u}\left(\theta, z_{o}, t\right)=\left.\stackrel{\circ}{u}(\bar{x}, t)\right|_{|\bar{x}|=R} & \text { in } L^{2}\left(\ell_{z_{o}, T}\right) ; & \\
\hat{v}\left(\theta, z_{o}, t\right)=\left.\stackrel{\circ}{v}(\bar{x}, t)\right|_{|\bar{x}|=R} & \text { in } L^{2}\left(\ell_{z_{o}, T}\right) ; \\
\hat{v}(\theta, z, t)=\left.v(\bar{x}, z, t)\right|_{|\bar{x}|=R} & \text { in } L^{2}\left(\ell_{z, T}\right), \quad \text { for all } z \in(0, H) .
\end{array}
$$

Since $z \rightarrow \hat{u}(\theta, z, t)$ is continuous in $L^{2}((0,2 \pi] \times(0, T]),(\mathrm{B} 3.4)$ implies

$$
\left.u\left(\bar{x}, z_{o}, t\right)\right|_{|\bar{x}|=R}=\left.\stackrel{\circ}{u}(\bar{x}, t)\right|_{|\bar{x}|=R} \quad \text { in } \quad L^{2}((0,2 \pi] \times(0, T]) .
$$

Therefore, while the interior limit $u(\bar{x}, z, t)$ and the limit $\stackrel{\circ}{u}(\bar{x}, t)$ in the special interdiscal space, might differ for $\{|\bar{x}|<R\}$, they coincide for $|\bar{x}|=R$. The limits $\hat{u}$ and $\hat{v}$ satisfy

$$
\begin{aligned}
\hat{u}_{t}-k_{u} \Delta_{S} \hat{u}= & -\left.\frac{\left(1-\theta_{o}\right) k_{u}}{\sigma \varepsilon_{o}} u_{\rho}\right|_{|\bar{x}|=R}-\left.\delta_{\ell_{z o}} \frac{v k_{u}}{\sigma} \stackrel{\circ}{u}_{\rho}\right|_{|\bar{x}|=R} \\
\hat{v}_{t}-k_{v} \Delta_{S} \hat{v}= & -\left.\frac{\left(1-\theta_{o}\right) k_{v}}{\sigma \varepsilon_{o}} v_{\rho}\right|_{|\bar{x}|=R}-\left.\delta_{\ell_{z o}} \frac{v k_{v}}{\sigma} \stackrel{\circ}{v}_{\rho}\right|_{|\bar{x}|=R} \quad \text { weakly in } S_{T} \\
& -\frac{1}{\sigma \varepsilon_{o}}\left\{g_{1}(\hat{v})-g_{2}(\hat{u})\right\} ; \\
\hat{u}_{z}, \hat{v}_{z}(\theta, 0, t)= & \hat{u}_{z}, \hat{v}_{z}(\theta, H, t)=0 \quad \forall \theta \in(0,2 \pi] \quad \forall t \in(0, T] .
\end{aligned}
$$

Both $\hat{u}$ and $\hat{v}$ are $C^{\infty}\left(S_{T}\right)$ except across $z=z_{o}$. The regularity requirements on $u$ and $\stackrel{\circ}{u}$ are not sufficient to ensure that $u_{\rho}$ and $\stackrel{\circ}{u}_{\rho}$ have traces in $L^{1}\left(S_{T}\right)$ and $L^{1}\left(\ell_{o, T}\right)$, respectively. In Section B4 we establish that this is the case. 


\section{B4. Weak form of the homogenized limit, regularity and uniqueness}

The functions $(u, \stackrel{\circ}{u}, \hat{u})$ and $(v, \stackrel{\circ}{v}, \hat{v})$ are in indicated regularity classes and satisfy

$$
\begin{aligned}
& \left(1-\theta_{o}\right)\left\{\begin{aligned}
\int_{\Omega_{o, T}}\left\{-u \varphi_{t}+k_{u} \nabla_{\bar{x}} u \cdot \nabla_{\bar{x}} \varphi\right\} d x d t-\int_{\Omega_{o}} u_{o} \varphi(x, 0) d x \\
+\int_{\Omega_{o, T}}\left(\gamma_{o} u-f_{1}(v)\right) \varphi d x d t
\end{aligned}\right\} \\
& +\sigma \varepsilon_{o}\left\{\int_{S_{T}}\left\{-\hat{u} \varphi_{t}+k_{u} \nabla_{S} \hat{u} \cdot \nabla_{S} \varphi\right\} d \eta d t-\int_{S} u_{o} \varphi(x, 0) d \eta\right\}_{\text {outer shell }} \\
& +v \varepsilon_{o}\left\{\begin{array}{l}
\int_{D_{R, T}}\left\{-\stackrel{\circ}{u} \varphi_{t}+k_{u} \nabla_{\bar{x}} \stackrel{\circ}{u} \cdot \nabla_{\bar{x}} \varphi\right\} d \bar{x} d t-\int_{D_{R}} u_{o} \varphi(x, 0) d x \\
+\int_{D_{R, T}}\left\{\gamma_{o} \stackrel{\circ}{u}-f_{1}(\stackrel{\circ}{v})\right\} \varphi d \bar{x} d t+\frac{1}{\nu \varepsilon_{o}} \int_{D_{R, T}} \stackrel{\circ}{u} f_{2}\left(\stackrel{\circ}{v}, \bar{x}, z_{o}, t\right) \varphi d \bar{x} d t
\end{array}\right\} \\
& =0 \text {, }
\end{aligned}
$$

for all testing functions $\varphi \in C^{1}\left(\bar{\Omega}_{o, T}\right)$ vanishing for $t=T$;

$$
\begin{aligned}
& \left(1-\theta_{o}\right)\left\{\int_{\Omega_{o, T}}\left\{-v \psi_{t}+k_{v} \nabla_{\bar{x}} v \cdot \nabla_{\bar{x}} \psi\right\} d x d t-\int_{\Omega_{o}} v_{o} \psi(x, 0) d x\right\}_{\text {interior }} \\
& +\sigma \varepsilon_{o}\left\{\int_{S_{T}}\left\{-\hat{v} \psi_{t}+k_{v} \nabla_{S} \hat{v} \cdot \nabla_{S} \psi\right\} d \eta d t-\int_{S} v_{o} \psi(x, 0) d x\right\} \\
& \left.+\frac{1}{\sigma \varepsilon_{o}} \int_{S_{T}}\left\{g_{1}(\hat{v})-g_{2}(\hat{u})\right\} \psi d \eta d t\right\}_{\text {outer shell }} \\
& +v \varepsilon_{o}\left\{\int_{D_{R, T}}\left\{-\stackrel{\circ}{v} \psi_{t}+k_{v} \nabla_{\bar{x}} \stackrel{\circ}{v} \cdot \nabla_{\bar{x}} \psi\right\} d \bar{x} d t-\int_{D_{R}} v_{o} \psi(x, 0) d \bar{x}\right\}_{\text {special }} \\
& =0,
\end{aligned}
$$

for all testing functions $\psi \in C^{1}\left(\bar{\Omega}_{o, T}\right)$ vanishing for $t=T$.

The same arguments of Section 6 may be applied here for the construction of suitable test functions. As a consequence, $u_{t}, \Delta_{\bar{x}} u \in L^{2}\left(\Omega_{o, T}\right), \hat{u}_{t}, \Delta_{S} \hat{u} \in L^{2}\left(S_{T}\right)$

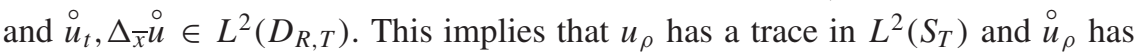
a trace in $L^{2}\left(\ell_{z_{o}}\right)$ so that (B3.5) have a pointwise meaning. By a bootstrap argument $\hat{u} \in C^{\infty}\left(S_{T}-\left\{z_{o}\right\}\right)$ and likewise $u \in C^{\infty}\left(\Omega_{o, T}-\left\{z_{o}\right\}\right)$.

Finally the uniqueness of Section 6.1 carries to this novel setting by minor variants.

\section{B5. Variants of the proof}

The calculation of these limits and the needed compactness follows the same lines of Sections 3-12 with some minor differences to which we point next. 
Statements of Proposition 3.1 hold for both $u_{\varepsilon}$ and $v_{\varepsilon}$. The only difference occurs in the proof of equi-boundedness of $v_{\varepsilon}$ and it is generated by the nonhomogeneous flux conditions in (B2.5). Such an upper bound is established by the barrier

$$
w(x, t)=C_{1} t+C_{2}\left(|\bar{x}|^{2}-R^{2}\right)^{2}+C_{3},
$$

where the positive constants $C_{1}, C_{2}, C_{3}$ are chosen so that

$$
a_{\varepsilon} w_{t}-k_{v} \operatorname{div} a_{\varepsilon} \nabla w \geq 0 \quad \text { in } \mathscr{D}^{\prime}\left(\widetilde{\Omega}_{\varepsilon, T}\right) .
$$

The upper bound is then established by an integral, weak form of the comparison principle, so that $0 \leq v_{\varepsilon} \leq w$. Energy and a time-regularity estimate of the type (3.2)-(3.3) for both $u_{\varepsilon}, v_{\varepsilon}$ and $\stackrel{\circ}{u}_{\varepsilon}, \stackrel{\circ}{v}_{\varepsilon}$, are standard.

The gradient estimates of Proposition 3.2 are first established for $v_{\varepsilon}$. The proof is simpler than the one in Section 8 , in view of the homogeneous flux conditions of $v_{\varepsilon}$ on $\partial I_{j}^{ \pm}$. Similar estimates for $u_{\varepsilon}$ are proven in all interdiscal spaces $I_{j}$ except the special interdiscal space $I_{j_{o}}$. In each of the $I_{j}, j \neq j_{o}$, the function $f\left(v_{\varepsilon}\right)$ now becomes a Lipschitz continuous function of $(x, z, t)$ so that the same arguments as in Section 8 apply.

A similar flow of arguments holds to prove an analogue of Proposition 3.3. One proves it first for $v_{\varepsilon}$ in view of its homogeneous flux conditions on $\partial I_{j}^{ \pm}$. Then the function $f\left(v_{\varepsilon}\right)$ appearing in the flux conditions for $u_{\varepsilon}$ becomes a Hölder continuous function of $z \in(0, H)$. The indicated modifications are reported in Section 12.1. It should be noted that the two interdiscal spaces $I_{j}=(0,0, h)+I_{i}$ in the statement of Proposition 3.3 and in the arguments of Sections 9-12 must both be taken to be different to $I_{j_{o}}$. Since the thickness of $I_{j_{o}}$ is $\nu \varepsilon<h$, the equi-Hölder continuity of $u_{\varepsilon}$ continues to hold across $I_{j_{o}}$.

\section{B5.1. The limit process}

The interior limit, as well as the limit on the outer shell, follow the argument in all similar ways to Sections 4-5. The new element here is the concentrated limit on the special interdiscal $I_{j_{o}}$. Set,

$$
\stackrel{\circ}{u}_{\varepsilon}(\bar{x}, t)=\frac{1}{\nu \varepsilon} \int_{\zeta_{2 j_{o}}}^{\zeta_{2 j_{o}+1}} u_{\varepsilon}(\bar{x}, \zeta, t) d \zeta ; \quad \stackrel{\circ}{v}_{\varepsilon}(\bar{x}, t)=\frac{1}{\nu \varepsilon} \int_{\zeta_{2 j_{o}}}^{\zeta_{2 j_{o}+1}} v_{\varepsilon}(\bar{x}, \zeta, t) d \zeta .
$$

By virtue of the energy estimates, subnets can be selected and relabelled with $\varepsilon$ such that $\left\{\stackrel{\circ}{u}_{\varepsilon}\right\},\left\{\stackrel{\circ}{v}_{\varepsilon}\right\} \rightarrow \stackrel{\circ}{u}, \stackrel{\circ}{v}$ strongly in $L^{2}\left(D_{R, T}\right)$ and $\left\{\nabla_{\bar{x}} \stackrel{\circ}{u}_{\varepsilon}\right\},\left\{\nabla_{\bar{x}} \stackrel{\circ}{v}_{\varepsilon}\right\} \rightarrow \nabla_{\bar{x}} \stackrel{\circ}{u}, \nabla_{\bar{x}} \stackrel{\circ}{v}$ weakly in $L^{2}\left(D_{R, T}\right)$. Moreover

$$
\left\{\frac{1}{\nu \varepsilon} \int_{\zeta_{2 j_{o}}}^{\zeta_{2 j_{o}+1}} v_{\varepsilon, z}(\bar{x}, \zeta, t) d \zeta\right\} \rightarrow \stackrel{\circ}{V_{z}} ; \quad\left\{\frac{1}{\nu \varepsilon} \int_{\zeta_{2 j_{o}}}^{\zeta_{2 j_{o}+1}} u_{\varepsilon, z}(\bar{x}, \zeta, t) d \zeta\right\} \rightarrow \stackrel{\circ}{U_{z}}
$$

weakly in $L^{2}\left(D_{R, T}\right)$. One identifies $\nabla_{\bar{x}} \stackrel{\circ}{u}$ and $\nabla_{\bar{x}} \stackrel{\circ}{v}$ as the weak gradient of $\stackrel{\circ}{u}$ and $\stackrel{\circ}{v}$ with respect to the $\bar{x}$-variables. However the two functions $\stackrel{\circ}{U}_{z}$ and $\stackrel{\circ}{V}_{z}$ are 
momentarily not identified. It will be shown later that they are both identically zero in $D_{R, T}$. By the energy estimates it follows that

$$
\int_{0}^{T} \int_{D_{R}} f\left(v_{\varepsilon}\right)\left(\zeta_{j_{o}}\right) d \bar{x} d t=\iint_{I_{j_{o}, T}} f\left(\stackrel{\circ}{v}_{\varepsilon}\right) d x d t+O(\varepsilon)
$$

A similar estimate holds for the integral over $\partial I_{j_{o}}^{+}$generated by the last of (B2.3). Proceeding as in Section 5, one writes down the weak formulation of (B2.1)-(B2.5) and divides the various integrals into the domains where $a_{\varepsilon}$ is constant. The two terms for $u_{\varepsilon}$ and $v_{\varepsilon}$ corresponding to the set $I_{j_{o}}$ are singled out and the limit is taken for $\varepsilon \rightarrow 0$. The limit of these terms corresponding to $u_{\varepsilon}$ is

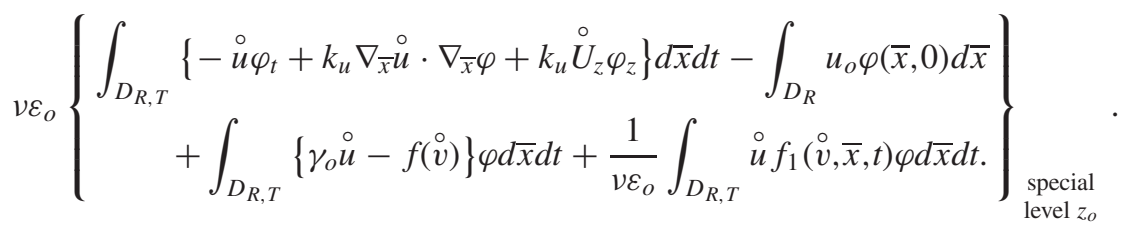

Similarly, the limit corresponding to $v_{\varepsilon}$ is,

$$
\nu \varepsilon_{o}\left\{\int_{D_{R, T}}\left\{-\stackrel{\circ}{v} \psi_{t}+k_{v} \nabla_{\bar{x}} \stackrel{\circ}{v} \cdot \nabla_{\bar{x}} \psi+k_{v} \stackrel{\circ}{V}_{z} \psi_{z}\right\} d \bar{x} d t-\int_{D_{R}} v_{o} \psi(\bar{x}, 0) d \bar{x}\right\}_{\substack{\text { special } \\ \text { level } z_{o}}} .
$$

One incorporates these two limits into the global weak formulations of (B2.1)(B2.5) and arrives at (B4.1)-(B4.2) with the extra two integrals involving $\stackrel{\circ}{U_{z}}$ and $\stackrel{\circ}{V_{z}}$. In this identity one chooses first a testing function $\varphi \in C^{\infty}\left(\mathbb{R}^{3} \times \mathbb{R}\right)$ independent of $z$, and then the test function $z \varphi$ for the previously chosen $\varphi$. Comparing the two expressions so obtained yields that $\stackrel{\circ}{U_{z}}=\stackrel{\circ}{V_{z}}=0$.

\section{Bibliography}

\section{Signal and photo-transduction}

[S1] Andreucci, D., Bisegna, P., DiBenedetto, E., Hamm, H.H.: Mathematical models of the dynamics of the second messengers in visual transduction: Homogenization and concentrated capacity. To appear in Biophys. J.

[S2] Calvert, P.D., Govardovskii, V.I., Krasnoperova, N., Andreson, R.E., Lem, J., Makino, C.L.: Membrane protein diffusion sets the speed of rod phototransduction. Nature 411, 90-94 (2001)

[S3] Gray-Keller, M., Denk, W., Shraiman, B., Detwiler, P.B.: Longitudinal Spread of Second Messenger Signals in Isolated Rod Outer Segments of Lizards. J. Physiol. 519, 679-692 (1999)

[S4] Heldin, C.-H., Purton, M. (eds.): Signal Transduction. Modular Texts in Molecular and Cell Biology 1. London: Chapman \& Hall 1996

[S5] Pugh Jr., E.N., Lamb, T.D.: Amplification and Kinetics of the Activation Steps in Phototransduction. Biochim. Biophys. Acta 1141, 111-149 (1993)

[S6] Pugh, E.N., Lamb, T.D.: Phototransduction in Vertebrate Rods and Cones: Molecular Mechanisms of Amplification, Recovery and Light Adaptation. Handbook of Biological Physics, Vol. 3, Chap. 5. Elsevier Science B.V. 2000

[S7] Stryer, L.: Visual Excitation and Recovery. J. Biol. Chem. 266, 10711-10714 (1991) 


\section{Homogenized limits}

[H1] Andreucci, D., Bisegna, P., DiBenedetto, E.: Homogenization and Concentrated Capacity in Reticular Almost Disconnected Structures. C. R. Acad. Sci., Paris, Sér. I, Math. 335, 329-332 (2002)

[H2] Bensoussan, A., Lions, J.L., Papanicolau, G., Asymptotic Analysis for Periodic Structures. New York: North-Holland 1978

[H3] Cioranescu, D., Donato, P.: On a Robin problem in perforated domains. Homogenization and applications to material sciences, 123-135. GAKUTO Int. Ser. Math. Sci. Appl. 9 (1997)

[H4] Cioranescu, D., Saint Jean Paulin, J.: Homogenization of reticulated structures. Appl. Math. Sciences 136, New York: Springer 1998

[H5] Oleinik, O.A., Shamaev, A.S., Yosifian, G.A.: Mathematical Problems in Elasticity and Homogenization. Studies in Mathematics and its Appl. 26, Amsterdam: North Holland 1992

[H6] Oleinik, O.A., Shaposhnikova, T.A.: On Homogenization problems for the Laplace operator in partially perforated domains with Neumann's condition on the boundary of cavities. Atti Accad. Naz. Lincei, Cl. Sci. Fis. Mat. Nat., IX Ser., Rend. Lincei, Mat. Appl. 6, 133-142 (1995)

[H7] Yosifian, G.A.: On some homogenization problems in perforated domains with nonlinear boundary conditions. Appl. Anal. 65, 257-288 (1997)

\section{Concentrated capacity}

[C1] Andreucci, D.: Existence and uniqueness of solutions to a concentrated capacity problem with change of phase. Eur. J. Appl. Math. 1, 339-351 (1990)

[C2] Ciarlet, Ph.G., Lods, V.: Asymptotic analysis of linearly elastic shells. I. Justification of membrane shell equations. Arch. Ration. Mech. Anal. 136, 119-161 (1996)

[C3] Colli, P., Rodrigues, J.F.: Diffusion through thin layers with high specific heat. Asymptotic Anal. 3, 249-263 (1990)

[C4] Erzhanov, R.Zh., Matsevityi, Yu.M., Sultangazin, U.M., Sheryshev, V.P.: Concentrated capacity in problems of thermophysics and micro-electronics, (Russian), 296pp. Kiev: Naukova Dumka 1992

[C5] Fasano, A., Primicerio, M., Rubinstein, L.: A model problem for heat conduction with a free boundary in a concentrated capacity. J. Inst. Math. Appl. 26, 327-347 (1980)

[C6] Magenes, E.: On a Stefan problem in a concentrated capacity. Nonlinear Anal. 217229. Quad. Sc. Norm. Sup., Pisa 1991

[C7] Magenes, E.: Stefan problems with a concentrated capacity. Boll. Unione Mat. Ital., Sez. B, Artic. Ric. Mat. (8) 1, 71-81 (1998)

[C8] Motygin, O.V., Nazarov, S.A.: Justification of the Kirchhoff hypotheses and error estimation for two-dimensional models of anisotropic and inhomogeneous plates, including laminated plates. IMA J. Appl. Math. 65, 1-28 (2000)

[C9] Savarè, G., Visintin, A.: Variational convergence of nonlinear diffusion equations: applications to concentrated capacity problems with change of phase. Atti Accad. Naz. Lincei Cl. Sci. Fis. Mat. Nat., IX Ser., Rend. Lincei, Mat. Appl. 8, 49-89 (1997)

\section{General references}

[G1] DiBenedetto, E.: Real Analysis. Boston: Birkhäuser 2002

[G2] Ladyzhenskaya, O.A., Solonnikov, V.A., Ural'tzeva, N.N.: Linear and Quasilinear Equations of Parabolic Type. Transl. Math. Monogr. 23. Providence, RI: Am. Math. Soc. 1968 